\title{
The time-dependent rheology of fermenting wheat flour dough: effects of salt and sugar
}

\author{
Mathieu Meerts ${ }^{\mathrm{a}} \quad$ Dries Vaes $^{\mathrm{a}} \quad$ Stefaan Botteldoorn $^{\mathrm{a}}$ \\ Christophe M. Courtin ${ }^{\mathrm{b}} \quad$ Ruth Cardinaels ${ }^{\mathrm{a}, \mathrm{c}} \quad$ Paula Moldenaers $^{\mathrm{a}}$
}

a Soft Matter, Rheology and Technology, Department of Chemical Engineering, KU Leuven, Celestijnenlaan 200F, Box 2424, BE 3001 Leuven, Belgium

b Laboratory of Food Chemistry and Biochemistry \& Leuven Food Science and Nutrition Research Center (LFoRCe), Department of Microbial and Molecular Systems, KU Leuven, Kasteelpark Arenberg 22, Box 2463, BE 3001 Leuven, Belgium

c Polymer Technology, Department of Mechanical Engineering, TU Eindhoven, Box 513, 5600 MB

Eindhoven, The Netherlands

Corresponding author: paula.moldenaers@cit.kuleuven.be 


\section{- Abstract}

The in-situ study of the linear viscoelastic behaviour of complex biological materials with changing volume, such as fermenting dough, poses great challenges to the rheologist. The aim of this study is to develop a new methodology involving a parallel-plate setup with an adjustable gap, to enable time-tracking of the dynamic moduli and density of fermenting dough. Frequency sweep snapshots at specific points in time were obtained in multiwave mode to reduce measurement times, and overfilling effects were taken into account by establishing a calibration curve with unfermented dough. The new test protocol allowed to distinguish the rheological impact of the $\mathrm{CO}_{2}$ gas from that of the other metabolites produced during fermentation. A further validation of the test protocol was achieved by studying the impact of sugar and salt on the fermentation kinetics, for which the results of the oscillatory tests were combined with gas production data obtained with a rheofermentometer.

Keywords Dough rheology; Fermenting dough; Multiwave frequency sweeps; Rheofermentometer

\section{Introduction}

It is widely recognised that the rheological properties of dough are closely related to the quality of the final product (Lee and Campanella, 2013). Consequently, the viscoelastic behaviour of wheat flour dough has been the subject of many rheological studies. However, the scope of most of these studies was restricted to unfermented dough, as in fermenting dough the sample dimensions and rheological properties vary significantly over time. Nonetheless, in view of the crucial importance of the fermentation stage in the breadmaking process, a proper rheological characterisation of fermenting dough is highly desirable. During fermentation, the cohesive dough mass (consisting of a viscoelastic network of gluten protein strands and starch granules) is transformed into a light, aerated structure by the action of yeast. Besides $\mathrm{CO}_{2}$, yeast produces many other metabolites, which may also affect the gluten-starch network (Jayaram et al., 2013). A distinction has to be made, therefore, between on the one hand the effect of the $\mathrm{CO}_{2}$ gas bubbles and on the other hand the impact of changes in the viscoelastic dough matrix due to fermentation. All in all, the impact of fermentation on the rheology of the dough matrix was found to be rather limited in degassed fermented dough (Meerts et al., 2018). Consequently, this effect will most likely be overshadowed by the impact of the $\mathrm{CO}_{2}$ gas bubbles if the dough is not degassed. To study the effect of the gas bubbles, the fermentation needs to be carried out inside the measurement device, as following fermentation the samples would otherwise become too fragile to allow a proper sample manipulation without collapse (Lee et al., 2004; Mert, 2008). The final gas volume of bread may well be over $70 \%$ of the loaf volume (Scanlon and Zghal, 2001). Several rheological studies of varying complexity have been conducted in situ during dough fermentation.

In a first type of tests, non-mechanical characterisation methods are used. Hoseney 
et al. (1979) devised a very simple spread test to determine the flowability of fermenting dough. Dough samples were allowed to stand freely, and their width over height ratio was measured over the course of $60 \mathrm{~min}$, starting after different fermentation times. Yeast activity was found to lead to less spreading of the dough. Elmehdi et al. (2003) and Lee et al. (2004) used ultrasonic techniques to study the viscoelastic nature of fermenting dough. The use of ultrasonic measurement techniques provides many advantages, as these tests are very sensitive and non-destructive. Ultrasound has therefore already been used extensively for the study of unfermented dough (for a recent review of this topic, the reader is referred to Koksel et al. (2016)). However, for fermenting dough, which is a very complex and heterogeneous material, the propagation of the ultrasonic waves proves to be difficult due to attenuation and scattering phenomena. As a result, ultrasonic techniques can only be used at the early stages of fermentation (Lee et al., 2004; Mert, 2008). Lower-frequency acoustic techniques at approx. $2 \mathrm{kHz}$ seem to be better suited to study the kinetics of the fermentation process (Skaf et al., 2009).

A second class of tests is formed by rheological methods that are based on mechanical characterisation. Kilborn and Preston (1982) modified the dough holder of an extensograph, a stretching device originally developed for the study of unfermented dough, which allowed them to study the extensional properties of fermenting dough. The fermenting dough had a far smaller failure strain and exhibited much less resistance to extension. Information on the linear viscoelastic behaviour of fermenting dough has been obtained by Mert (2008) and Lee and Campanella (2013), by applying oscillatory flows in compression and extension, respectively. In both studies the gap between the parallel plates was held constant; in the study conducted by Mert (2008) excess sample material was trimmed at regular time intervals (every $10 \mathrm{~min}$ ). These studies revealed a clear loss of elasticity in fermenting dough (Lee and Campanella, 2013) and also highlighted the important effect of temperature on the fermentation kinetics and final dough stiffness (Mert, 2008).

The aim of the present study is to develop a new mechanical characterisation protocol that allows us to probe the linear viscoelastic nature of fermenting dough in-situ and time-resolved, using a setup of Mora et al. (1991) developed to study urethane foaming as a starting point. The fixture used in the present study equally consists of two parallel plates, enclosed in a large cup, but the gap between the plates can be adjusted, based on the reading of the normal force, to accommodate the significant volume expansion of the fermenting dough sample without damaging the material. Other studies have shown that restrictions (either in the horizontal or vertical direction) do affect the leavening potential of the dough (Elmehdi et al., 2007; Chevallier et al., 2012). Values for the dynamic moduli are obtained over approximately three decades of frequency; however, in view of the highly dynamic nature of the fermentation process, the frequency sweeps need to be carried out in multiwave mode to reduce the measurement time. As was the case in the study conducted by Mora et al. (1991), edge effects are accounted for by a calibration curve. Since the rheological response of fermenting dough is determined first and foremost by the (expanding) $\mathrm{CO}_{2}$ gas bubbles, the rheological experiments are 
complemented by an analysis of the dough density (based on the time evolution of the measurement gap in the rheometer) and an analysis of the gas production data (as obtained with a commercially available rheofermentometer (Czuchajowska and Pomeranz, 1993)). Apart from the changes in the viscoelastic nature of the dough during fermentation, this combined approach yields information on the fermentation kinetics, the volume expansion, the amount of gas produced by the yeast and the fraction that is retained within the dough matrix. Finally, salt $(\mathrm{NaCl})$ and sugar (sucrose) are known to alter the rheology of the gluten-starch network (and hence its gas-holding capacity) as well as the yeast activity, and are often added to control the fermentation process. With the developed methodology, the impact of these constituents on the breadmaking potential of wheat flour dough is investigated in both the unfermented and the fermenting state. The obtained insights can help to achieve a better understanding of the fermentation process and allow for a further optimisation.

\section{Materials and methods}

\subsection{Dough preparation}

The Bison flour used in this study is a weak wheat flour, obtained from Dossche Mills (Deinze, Belgium). The protein content was determined with an automated Dumas protein analysis system (EAS, VarioMax N/CN, Elt, Gouda, The Netherlands) following an adaptation of the AOAC method 990.03 (AOAC International, 1995), and equalled $12.4 \mathrm{wt} \%$ on a dry matter basis. The moisture content of the Bison flour was $12.7 \mathrm{wt} \%$, according to AACC method 44-19.01 (AACC International, 2000).

Following the common practice in cereal science, the optimal amount of water to be added and the optimal mixing time were determined with a farinograph (Brabender, Duisburg, Germany) and a mixograph (National Manufacturing, Lincoln, USA) in accordance with AACC methods 54-40.02 and 54-21.02, respectively (AACC International, 2000). Dough samples consisted of $10 \mathrm{~g}$ flour (on $14 \mathrm{wt} \%$ moisture base) with $5.3 \mathrm{wt} \%$ of compressed baker's yeast (Algist Bruggeman, Ghent, Belgium), $1.5 \mathrm{wt} \%$ $\mathrm{NaCl}, 6 \mathrm{wt} \%$ sucrose, and $5.4 \mathrm{ml}$ of water (AACC method 10-10.03). In some cases, the salt or sugar component was omitted from the dough recipe to study the effect of these additives on the rheology of unfermented and fermenting dough. All ingredients were mixed in a $10 \mathrm{~g}$ pin bowl mixer (National Manufacturing) for $3 \mathrm{~min} 30 \mathrm{~s}$ to reach the optimal consistency. After mixing, the dough samples were shaped with a pasta machine to obtain a quasi-cylindrical shape with a height of approx. $4-5 \mathrm{~mm}$ and a diameter of at least $40 \mathrm{~mm}$. Subsequently, the dough samples were loaded in the rheometer. As a reference, dough without yeast was prepared in the same way. To investigate the impact of salt and sugar, yeast-free doughs were also prepared according to a slightly different protocol: after mixing and sheeting, the unfermented dough samples were rested for 6 hours in a climate chamber (HPP 749 , Memmert, Schwabach, Germany) held at $32{ }^{\circ} \mathrm{C}$ and at a relative humidity of $75 \%$. Subsequently, the samples were sheeted again, and 
finally loaded in the rheometer.

\subsection{Fundamental rheological methods}

Small amplitude oscillatory shear (SAOS) tests were performed on a stress-controlled MCR501 rheometer (Anton Paar, Graz, Austria), using a $40 \mathrm{~mm}$ parallel plate geometry. To prevent dehydration of the dough samples, a solvent trap combined with wet cotton wool was used. Slip effects were eliminated by coating the top and bottom plates with sandpaper. Rheological experiments were performed at $32{ }^{\circ} \mathrm{C}$, as wheat flour doughs are generally fermented at, or slightly below this temperature (Cauvain and Young, 2007). After being loaded in the rheometer, the fermenting dough sample was subjected to a time sweep (angular frequency $\omega=1 \mathrm{rad} / \mathrm{s}$; linear strain amplitude $\gamma_{0}=0.06 \%$; linearity was verified) for two hours to track the rheological changes during fermentation. To accommodate the volume expansion, the measurement gap was gradually increased during fermentation based on the reading of the normal force (see section 3.1 for a detailed discussion of the measurement protocol). At specific points in time (15 min, $30 \mathrm{~min}, 60 \mathrm{~min}$ and $90 \mathrm{~min}$ ), frequency sweeps were performed. The frequency sweeps were carried out in multiwave mode, in order to reduce the measurement time.

From a structural point of view, dough is a specific example of a foam, consisting of $\mathrm{CO}_{2}$ gas bubbles dispersed in a viscoelastic gluten-starch matrix. Mora et al. (1991) conceived a flooded parallel plate fixture to track the modulus build-up during reactive urethane foaming, which was later improved by Neff and Macosko (1996). In this setup the expanding polymer sample was allowed to protrude from the gap between the top and bottom plates, which was kept constant. The excess material was collected in a disposable cup attached to the bottom plate. To account for the contribution of the edges and the cup, the setup was calibrated using shaving cream as test material, resulting in a calibration factor of about 3.3 for the dynamic moduli (Neff and Macosko, 1996). Following the approach outlined by Mora et al. (1991) and Neff and Macosko (1996), a homemade cup containing the bottom plate was used to allow dough expansion in a controlled environment during the rheological tests (see Fig. 1). The aluminium bottom plate had an inner diameter of $115 \mathrm{~mm}$ and a height of $12.5 \mathrm{~mm}$, and could be attached to the commercial peltier bottom plate produced by Anton Paar, thus enabling an accurate temperature control. To provide sufficient space for the expanding dough sample, two half rings made of PMMA were placed on top of the aluminium bottom plate. These PMMA rings were almost see-through to allow a visual inspection of the rising dough. The height of the PMMA rings was $20 \mathrm{~mm}$, whereas the inner diameter varied from $80 \mathrm{~mm}$ at the top (which is slightly smaller than the inner diameter of the large solvent trap, which is $85 \mathrm{~mm}$ ), to $100 \mathrm{~mm}$ at the bottom (to accommodate the radial expansion of the dough sample).

Dynamic measurements were also performed on unfermented dough, to investigate the impact of variations in the salt and sugar content. After a time sweep of $5 \mathrm{~min}$, a regular frequency sweep in the linear region was performed at fixed gap. 
All dynamic measurements were conducted at least in triplicate on separately prepared batches of dough (with the average values being shown). Good reproducibility was obtained with relative standard deviations between $3 \%$ and $16 \%$ for unfermented as well as fermenting dough. To determine whether the observed differences between dough samples prepared with different sugar or salt concentrations were statistically significant $(P<0.05)$, the two-tailed unpaired Welch's $t$-test was used.

The extensional behaviour of dough is known to be dominated by the gluten network, whereas linear oscillatory tests tend to probe the response of both the gluten network and the starch granules (Amemiya and Menjivar, 1992). Extensional tests thus constitute a valuable addition to the oscillatory shear tests, as the gas-holding capacity of fermenting dough is determined first and foremost by the gluten network. An Extensional Viscosity Fixture (EVF) mounted on a strain-controlled ARES-G2 rheometer (TA Instruments, New Castle, USA) was used to study the extensional behaviour of unfermented dough and the impact of sugar and salt thereon. The sugar- and/or saltsupplemented unfermented dough samples were treated in a similar way as described above for the dynamic measurements, but the samples were allowed an additional resting time of $15 \mathrm{~min}$ between the second sheeting action and the loading step. The extensional tests were started directly after loading. The EVF setup consists of two drums to which the dough strand can be attached. Extension at a constant rate $\left(0.1 \mathrm{~s}^{-1}\right.$ in our case) is obtained as one drum remains stationary and the other moves in a circular orbit around it whilst also rotating around its own axis. As a measure for deformation, we used the Hencky strain $\epsilon(t)[-]$ :

$$
\epsilon(t)=\ln \left(\frac{L(t)}{L_{0}}\right)
$$

Here, $L_{0}$ represents the initial length of the dough strand, and $L(t)$ equals the actual length during extension. The transient extensional viscosity $\eta_{e}^{+}(\epsilon)[\mathrm{Pa} s]$ can be defined as:

$$
\eta_{e}^{+}(\epsilon)=\frac{\sigma_{11}(\epsilon)}{\dot{\epsilon}}
$$

In this expression $\sigma_{11}(\epsilon)$ corresponds to the longitudinal stress $[\mathrm{Pa}]$ calculated from the torque registered by the transducer of the rheometer. The maximum achievable strain with the EVF setup was limited to approx. 2.9; it was therefore not possible to determine the extensibility of the dough, as the samples could not be stretched until failure. The extensional viscosity curves are the average of $10-25$ measurements on 2 - 4 separately prepared batches. As these measurements are not all independent, the pooled standard deviation $s_{p}$ was used to determine the data variability (McNaught and Wilkinson, 1997):

$$
s_{p}=\sqrt{\frac{\sum_{i}\left[\left(n_{i}-1\right) s_{i}^{2}\right]}{\sum_{i}\left[n_{i}\right]-N}}
$$


In this expression, $s_{i}$ represents the standard deviation for the measurements of batch i. The number of measurements in each batch is denoted by $n_{i}$, and the total number of batches is given by $N$. The relative standard deviation varied between $10 \%$ and $24 \%$. More information on the use of the EVF setup for dough measurements can be found in Meerts et al. (2017a).

\subsection{Rheofermentometer analysis}

A rheofermentometer F3 (Chopin Technologies, Villeneuve-la-Garenne, France) was used to monitor the gas production by the yeast cells in the dough over the course of two hours, based on the procedure explained by Czuchajowska and Pomeranz (1993). Dough samples of $100 \mathrm{~g}$ were prepared in a $100 \mathrm{~g}$ pin bowl mixer (National Manufacturing) in a similar way as described above and placed in the basket of the device which was held at $32^{\circ} \mathrm{C}$. The dough sample was spread out evenly over the bottom of the basket, and subsequently covered by a piston with a mass of $250 \mathrm{~g}$ and a diameter of $11 \mathrm{~cm}$. Typically, a weight of $2 \mathrm{~kg}$ is used in rheofermentometer tests, but to mimic the configuration of the rheometer as closely as possible (and hence minimise expansion restrictions), the minimum available piston weight of $250 \mathrm{~g}$ was used. The piston thus exerted a pressure of about $260 \mathrm{~Pa}$ on the fermenting dough, which is lower than the pressure exerted by the top plate in the rheometer at the start of fermentation (ca. $400 \mathrm{~Pa}$ ) but higher than the pressure at the end of fermentation (as a normal force value of $0.05 \mathrm{~N}$ corresponds to a pressure of ca. $40 \mathrm{~Pa}$ with the parallel-plate setup, see also section 3.1.1). The upward movement of the piston is recorded by a displacement sensor which allows to determine the dough volume expansion and to construct a dough development curve. The rheofermentometer equally records the total gas production via a pressure sensor. In addition, the device is able to distinguish between the amount of gas retained within the dough and the amount of gas released by the dough, by performing two pressure measurement cycles in series, using a pneumatic circuit. The pneumatic circuit connects the basket in which the dough is fermenting to the pressure sensor via two parallel connections. The first connection directly links the basket to the pressure sensor, and allows to determine the total gas production. The second connection additionally includes a soda lime cartridge, which can absorb $\mathrm{CO}_{2}$. When the first connection is closed and the second connection opened, the released $\mathrm{CO}_{2}$ is absorbed by the soda lime cartridge, and the pressure increase caused by the rising dough without the contribution of the released $\mathrm{CO}_{2}$ can be obtained. The pressure difference between the two cycles yields the amount of gas which escaped from the dough. The gaseous release curves are typically characterised by the following set of parameters: the maximum fermentation rate $F_{m}$ $\left[\mathrm{ml} \mathrm{CO}_{2} / \mathrm{min}\right]$, which corresponds to the maximum in the gaseous release curves; the time $t_{m}[\mathrm{~h}]$ at which this maximum is reached; the total amount of gas produced $V_{t o t}$ $[\mathrm{ml}]$; the amount of gas retained within the dough $V_{\text {ret }}[\mathrm{ml}]$; the amount of gas released by the dough $V_{\text {rel }}[\mathrm{ml}]$, which equals $V_{\text {tot }}-V_{\text {ret }}$; and the time $t_{x}[\mathrm{~h}]$ at which the dough starts to release gas. 


\section{Results and discussion}

\subsection{Development of the measurement protocol}

As a consequence of the $\mathrm{CO}_{2}$ produced by yeast, the fermenting dough sample will experience a gradual but significant volume expansion. In order to obtain reliable oscillatory data, the gap between the two parallel plates of the rheometer should be adjusted accordingly, thus minimising the restrictions felt by the dough during expansion. The normal force transducer of the rheometer allows to track the pressure buildup within the dough, and can be used to steer the concomitant evolution of the measurement gap.

\subsubsection{Normal force protocol}

Keeping the normal force constant for the entire duration of the experiment turned out not to be feasible: high values $(1 \mathrm{~N})$ eventually led to a collapse of the fermenting dough samples as their structure progressively weakened with increasing gas volume fraction, whereas low values $(0.05 \mathrm{~N})$ did not suffice to establish a good contact between the dough sample and the top plate at the start of the experiment. Hence, the normal force was varied linearly from $0.5 \mathrm{~N}$ to $0.05 \mathrm{~N}$ for the first $30 \mathrm{~min}$, and subsequently held constant at $0.05 \mathrm{~N}$ (which is close to the detection limit of the MCR501 rheometer) for the remaining $90 \mathrm{~min}$. The storage modulus $G^{\prime}(t)$ and loss modulus $G^{\prime \prime}(t)$ at $\omega=$ $1 \mathrm{rad} / \mathrm{s}$ and $\gamma_{0}=0.06 \%$ were recorded every $30 \mathrm{~s}$. Fig. 2a shows the evolution of the dynamic moduli over time during fermentation. The moduli tend to decrease strongly as fermentation progresses. The phase angle $\delta(t)$ decreases slightly (from $30^{\circ}$ to $22^{\circ}$ ), indicating that relatively speaking the viscous response is reduced to a greater extent by the fermentation than the elastic response. The corresponding evolution in dough height is given by Fig. $2 \mathrm{~b}$. The adopted normal force profile gives rise to a very smooth increase in the measurement gap $H(t)$. The transition between the two regimes in the normal force profile was arbitrarily set at $30 \mathrm{~min}$. Other times ( $15 \mathrm{~min}$ and $60 \mathrm{~min}$ ) were tried as well, and it was found that the time span over which the normal force is varied has no significant effect on the time evolution of the dynamic moduli or the dough expansion (results not shown). Besides using linear profiles, the normal force was also varied in a stepwise manner from 0.5 to $0.05 \mathrm{~N}$ over the course of $30 \mathrm{~min}$, dropping by $0.09 \mathrm{~N}$ every 6 min. Apart from some very small peaks in $G^{\prime}(t)$ at each stepwise reduction in normal force, the transformation from a linear to a stepwise normal force profile did not lead to substantial changes in the dynamic moduli or the dough height (results not shown). These observations indicate that the applied normal force profile does not fundamentally affect the dynamics of the fermentation process under the investigated conditions. The measurements in Fig. 2 were performed on three different samples prepared on different days. Despite the intrinsic fragility of the fermenting dough samples, the rheological data obtained with the parallel-plate setup with expanding gap turned out to be very reproducible. 


\subsubsection{Multiwave frequency sweeps}

Besides the time dependency, the frequency dependency of the dynamic moduli might also yield insight in the dynamics of the fermentation process. Therefore, frequency sweeps were carried out as well on the fermenting dough samples, at specific moments in time (i.e. $15 \mathrm{~min}, 30 \mathrm{~min}, 60 \mathrm{~min}$ and $90 \mathrm{~min}$ after the start of the time sweep). In these experiments the linear normal force profile was adjusted to reach the constant value of $0.05 \mathrm{~N}$ already after $15 \mathrm{~min}$, so that the normal force would remain constant during each frequency sweep. As the dynamic moduli are changing over time, the measurement time for each frequency sweep needs to be as short as possible, in order to avoid significant evolution of the dough rheology during the frequency sweep. The measurement time can be reduced by limiting the number of frequency values probed in each measurement, and by focussing predominantly on the higher frequencies. In addition, one can choose to limit the number of cycles used by the MCR501 rheometer to determine the dynamic moduli. In the rheometer software the default value for the number of cycles is approx. 4 cycles, whereas the minimum allowed value is 3 . Finally, the frequency sweeps were carried out in multiwave mode, which means that several frequencies were probed simultaneously, instead of sequentially as in a regular frequency sweep. The MCR501 is capable of performing multiwave tests, but being a stress-controlled device, it can only do so in stress-controlled mode. A maximum of three frequencies can be applied simultaneously, and for the higher-order oscillations only integer multiples of the base frequencies are accepted by the rheometer software. To cover a sufficiently wide frequency range, three base frequencies were used $(0.215 \mathrm{rad} / \mathrm{s}, 0.464 \mathrm{rad} / \mathrm{s}$ and $1 \mathrm{rad} / \mathrm{s}$ ), and each base frequency was multiplied by a factor of 10 and 100. The data obtained at $\omega=100 \mathrm{rad} / \mathrm{s}$ were discarded, as for these data we could not be certain that the applied deformation signal was in phase over the entire sample height because of the large measurement gap (see section 3.1.5). With three frequencies being probed simultaneously, the stress amplitude $\sigma_{\text {tot }}$ of the combined signal equals three times the stress amplitude $\sigma_{0}$ of each constituent signal (provided that each constituent signal has the same stress amplitude). The linearity limit of (unfermented) dough was found to increase slightly with increasing angular frequency; at the lowest frequency probed ( $\omega$ $=0.1 \mathrm{rad} / \mathrm{s}$ ), its value equalled about $3 \mathrm{~Pa}$ (results not shown). Consequently, each frequency was probed with a stress amplitude $\sigma_{0}=0.5 \mathrm{~Pa}$, such that the resulting stress amplitude $\sigma_{\text {tot }}$ still fell within the linear region. With these settings, the measurement time for each frequency sweep could be reduced from $15 \mathrm{~min}$ to $3 \mathrm{~min}$, approximately. Within this short time frame, $G^{\prime}$ declined by approx. $19 \%$ at most (i.e. during the first sampling of the frequency sweep, 15 min after fermentation was started). While this error is larger than the statistical uncertainty on the rheological data (see Materials and methods), on a log-log plot spanning several decades such a change is still barely noticeable and consequently does not alter the shape of the frequency sweep significantly. Excellent agreement between regular frequency sweeps and multiwave frequency sweeps performed on unfermented dough indicated that the multiwave approach yielded reliable data (results not shown, but relative deviations were at most $7 \%$ for $G^{\prime}$ and $2 \%$ for $G^{\prime \prime}$ ). 


\subsubsection{Calibration curve for $G^{\prime}(t)$ and $G^{\prime \prime}(t)$ to correct for overfilling}

During fermentation the dough expands not only in the vertical direction, but also in the horizontal direction. At the moment of loading, the dough sample is trimmed to match the diameter of the top plate, but excess dough will quickly start to appear as a consequence of the dough's horizontal expansion. This excess dough around the parallel-plate geometry will exert an extra torque on the dough material between the plates. Hence, the initial stress-free boundary condition along the sample edges will be violated, leading to an overestimation of the actual moduli. These edge effects can be fairly significant, and should be accounted for. To estimate the contribution of the dough edges to the value of the dynamic moduli, an attempt was made to trim a set of fermenting dough samples after specific fermentation times, and to register the factor by which the dynamic moduli drop as a result of the trimming action. Shearing effects were limited by the use of a sharp razor blade. However, this calibration approach turned out not to be feasible: without the support of the sample edges the fermenting dough samples tended to collapse as their aerated structure proved to be extremely fragile.

To obtain an indication of the impact overfilling has on the dynamic moduli, unfermented dough was used instead of fermenting dough, thereby circumventing the issue of sample fragility. The contribution of the dough edges to the value of the dynamic moduli depends on the sample height $H$, the sample diameter $D$ and on the overfilling volume. Information on the evolution of $H$ and $D$ during fermentation was obtained from the trimming experiments on fermenting dough (see above). Subsequently, five values for $H$ were selected: $6.4 \mathrm{~mm}, 7.3 \mathrm{~mm}, 10.4 \mathrm{~mm}, 11.5 \mathrm{~mm}$ and $13 \mathrm{~mm}$. The corresponding values for $D$ were $42.2 \mathrm{~mm}, 44 \mathrm{~mm}, 50 \mathrm{~mm}, 52.5 \mathrm{~mm}$ and $55 \mathrm{~mm}$, respectively. Appropriate moulds were made to create samples of unfermented dough with the desired cylindrical shapes. The untrimmed samples were loaded in the rheometer and subsequently subjected to a time sweep of $15 \mathrm{~min}\left(\omega=1 \mathrm{rad} / \mathrm{s}, \gamma_{0}=0.06 \%\right)$ followed by a multiwave frequency sweep (see section 3.1.2). Each $(H, D)$ pair was tested in threefold, using separately prepared dough batches. With the known $G^{\prime}$ and $G^{\prime \prime}$ values of unfermented dough, which are independent of the sample size, the five measurement points thus obtained allowed to construct a calibration curve, from which the scale factors for $G^{\prime}$ and $G^{\prime \prime}$ could be obtained as a function of $(H, D)$. The scale factors for $G^{\prime}$ and $G^{\prime \prime}$ turned out to be nearly identical, and were found not to depend on frequency. The calibration curve for $G^{\prime}$ is given by the following expression:

$$
\text { scale factor } G^{\prime}=0.38+5.4 \cdot \exp \left(-2.2 \cdot H / H_{0}\right)
$$

with $H_{0}$ the initial sample height at the start of the rheological measurement, and $H$ the actual sample height. The calibration curve for $G^{\prime}$ is also depicted in Fig. 3a, along with the original and rescaled dynamic moduli for fermenting dough. The rescaled values were obtained by multiplying the original values for the dynamic moduli with the corresponding scale factors provided by the calibration curve as a function of $H / H_{0}$. Fig. 3a shows that the drop in the dynamic moduli as a result of fermentation becomes even more pronounced when the effect of overfilling is taken into account. The calibra- 
tion curve thus not only corrects for the absolute value of the dynamic moduli, but also reveals the true kinetics of the fermentation process. Care has to be taken, however, as the calibration curve was established for unfermented dough, so for fermenting dough the excess material at the edges might exert a somewhat different effect on the dynamic moduli. A full study of the effect of the material viscoelastic properties on the implications of overfilling is however beyond the scope of the present work.

\subsubsection{Calibration curve for $V(t)$ to include radial expansion}

As the fermenting dough sample in the rheometer is allowed to expand freely in the radial direction, information on the sample height $H(t)$ does not suffice to track the change in the sample volume $V(t)$ over time. When approximating the shape of the fermenting dough sample by a cylinder, knowledge of the time evolution of the sample diameter $D(t)$, in addition to $H(t)$, is sufficient to establish $V(t)$. The trimming experiments on fermenting dough (see section 3.1.3) indicated that the sample diameter $D(t)$ did not exhibit the same evolution as the sample height $H(t)$. Yet, when rescaling the sample diameter and height with the corresponding initial values $\left(D_{0}\right.$ and $\left.H_{0}\right)$, the relation between $D(t) / D_{0}$ and $H(t) / H_{0}$ turned out to be nearly linear, as shown in Fig. 3b:

$$
\frac{D(t)}{D_{0}} \approx 0.16 \frac{H(t)}{H_{0}}+0.84
$$

The assumption of a cylindrical sample shape leads to the following relation between the relative increase in sample volume $V(t) / V_{0}$ and the relative increase in sample height and diameter:

$$
\frac{V(t)}{V_{0}}=\left(\frac{D(t)}{D_{0}}\right)^{2} \cdot \frac{H(t)}{H_{0}} \approx\left(0.16 \frac{H(t)}{H_{0}}+0.84\right)^{2} \cdot \frac{H(t)}{H_{0}}=f\left(\frac{H(t)}{H_{0}}\right)
$$

Taking the radial expansion into account, the increase in sample height can be translated into an increase in sample volume, as shown in Fig. 3b. The sigmoidal shape of the expansion profile for fermenting dough has already been noted in several other studies (Ito et al., 1992; Elmehdi et al., 2003; Romano et al., 2007). The initial lag phase reflects the time needed for the yeast to adapt to its new environment and for the $\mathrm{CO}_{2}$ to diffuse to the air nuclei upon saturation of the aqueous phase in dough (Romano et al., 2007; Mert, 2008). The $\mathrm{CO}_{2}$ production by yeast does not result in the creation of new gas bubbles; instead the produced $\mathrm{CO}_{2}$ will only lead to an increase in size of gas bubbles that were already created by the entrapment of air in the mixing stage (Baker and Mize, 1941). Eventually, a balance is reached between the amount of gas produced by the yeast and the amount of gas released by the dough, after which the volume of the fermenting dough will no longer increase, as evidenced by Fig. 3b (Chevallier et al., 2012). The final dough volume is almost 4.4 times larger than the initial dough volume before the start of fermentation. Similar increases in dough volume have been reported elsewhere (Romano et al., 2007; Chevallier et al., 2012). 


\subsubsection{Wave propagation in large gap rheometry}

Finally, it is important to note that the use of large measurement gaps (about 10 $12 \mathrm{~mm}$ ) at the end of the two-hour fermentation period might entail the risk of creating non-homogeneous flow fields within the dough sample, which would confound the rheological data. Ewoldt et al. (2015) mention that the wavelength $l[\mathrm{~m}]$ of the applied oscillatory deformation should equal at least 10 times the measurement gap for the entire sample to be "in phase" with the applied deformation. According to Ewoldt et al. (2015), the wavelength $l$ of the oscillatory deformation can be determined as:

$$
l=\frac{1}{\cos (\delta / 2)} \cdot \sqrt{\frac{\left|G^{*}\right|}{\rho}} \cdot \frac{2 \pi}{\omega}
$$

In this expression, $\delta$ stands for the phase angle $\left[{ }^{\circ}\right], G^{*}$ is the complex modulus $[\mathrm{Pa}]$, and $\rho$ the dough density $\left[\mathrm{kg} / \mathrm{m}^{3}\right]$. It turns out that the minimum wavelength $l$ is obtained in the frequency sweep determined after $60 \mathrm{~min}$ of fermentation and for the highest angular frequency $(\omega=46.4 \mathrm{rad} / \mathrm{s})$. Taking into account the scale factor obtained from the calibration curve depicted in Fig. 3a, $\left|G^{*}(\omega=46.4 \mathrm{rad} / \mathrm{s})\right|$ is about $1040 \mathrm{~Pa}$. At that instant the dough density has already dropped by a factor of about 4 . The initial dough density was determined as follows: after mixing, three dough samples were sheeted with the pasta machine to obtain a layer thickness of $4 \mathrm{~mm}$, and subsequently cylindrical discs of $55 \mathrm{~mm}$ in diameter were created with a mould. Finally, the mass of the moulded dough samples was determined. The initial dough density amounted to approx. $1240 \pm 55 \mathrm{~kg} / \mathrm{m}^{3}$, so the actual dough density after $60 \mathrm{~min}$ of fermentation would be close to $300 \mathrm{~kg} / \mathrm{m}^{3}$. Combining these results yields a minimum value of $250 \mathrm{~mm}$ for the wavelength $l$, which implies that the measurement gap should be less than $25 \mathrm{~mm}$ in order to obtain reliable data over the entire frequency range. In our experiments the measurement gap reaches values of $14 \mathrm{~mm}$ at most. Hence, all data presented in this study are free from errors due to wave propagation issues. However, a similar calculation for the data at $\omega=100 \mathrm{rad} / \mathrm{s}$ shows that these data cannot be used.

\subsection{The time-dependent rheology of fermenting dough}

The previous discussion revealed that the developed methodology can be trusted to yield accurate and reproducible rheological data. Following calibration, information on the dynamic moduli and the sample volume can be obtained simultaneously in a single rheological experiment. In the second part of this study, the rheological setup with expanding gap will be used to investigate the impact of yeast fermentation on the linear viscoelastic behaviour of fermenting dough. Fig. 4a compares the time evolution of the normalised dynamic moduli of fermenting dough corrected for overfilling effects (as obtained from Fig. 3a) with that of the sample density (as derived from the $V(t) / V_{0}$ data depicted in Fig. 3b). As fermentation progresses, the dynamic moduli of the fermenting dough decrease substantially. This softening effect of fermentation was already noted in earlier studies (Lee et al., 2004; Lee and Campanella, 2013), and is typically attributed 
to the expanding $\mathrm{CO}_{2}$ gas bubbles (Verheyen et al., 2014). It is important to note that even though the time evolution of the dough density and the dynamic moduli is very similar, the kinetics are not exactly the same. Compared to the dough density $\rho(t)$, the lag phase is much shorter (in fact practically absent) for $G^{\prime}(t)$ and $G^{\prime \prime}(t)$. The storage modulus $G^{\prime}(t)$ also reaches the midpoint between its initial and final relative value approx. $300 \mathrm{~s}$ earlier than the dough density $\rho(t)$. In ultrasonic measurements, Elmehdi et al. (2003) equally observed that at the initial stage of the fermentation process, the ultrasonic velocity was already declining before the dough density changed markedly. This discrepancy indicates that the yeast activity affects the dough rheology in several ways. Besides $\mathrm{CO}_{2}$, yeast produces many other metabolites, such as ethanol, succinic acid and glycerol (Jayaram et al., 2013, 2014a,b; Aslankoohi et al., 2015). Whereas the expanding $\mathrm{CO}_{2}$ gas cells substantially lower the dough density, the other metabolites additionally exert a (limited) softening effect on the dough matrix (Meerts et al., 2018), and may thus intensify the destabilising impact of the $\mathrm{CO}_{2}$ gas bubbles. Finally, due to the increased dough acidity, proteolytic enzymes exhibit increased activity in fermenting dough, thereby reducing the elasticity of the dough matrix to an even greater extent (Verheyen et al., 2014). All these factors combined may explain why the decrease of the dynamic moduli does not exactly follow the time evolution of the dough density.

The frequency dependence of the dynamic moduli and the phase angle is illustrated in Fig. 4b for unfermented dough and for fermenting dough after $15 \mathrm{~min}$ and $90 \mathrm{~min}$ of fermentation, respectively. To arrive at this figure, the unfermented dough was subjected to the same treatment as the fermenting dough (i.e. sample loading was done immediately after mixing), but evidently the unfermented dough did not contain any yeast. Fig. 4b shows that as fermentation progresses the dynamic moduli drop significantly over the entire frequency range. Surprisingly, already after $15 \mathrm{~min}$ of fermentation, a considerable difference appears in the values for the phase angle $\delta$ between unfermented and fermenting dough, predominantly at the lower frequencies. As the fermentation progresses, the differences in phase angle tend to become smaller again. Frequency sweeps conducted on dough that was fermented for 6 hours and from which the $\mathrm{CO}_{2}$ gas bubbles had been removed by sheeting, also pointed out that the moduli of the dough matrix are lowered predominantly at low frequencies as a consequence of fermentation (Meerts et al., 2018). It thus seems that initially some metabolites (other than $\mathrm{CO}_{2}$ ) released by yeast to the fermenting dough affect the viscoelasticity of the dough matrix, leading to a sudden drop in the dynamic moduli of the fermenting dough at low frequencies. Yet, as the fermentation continues, $\mathrm{CO}_{2}$ gas bubbles are formed, and the accompanying decrease in dough density will dominate all other effects; hence, the drop in the dynamic moduli is extended over the entire frequency range. At present, it is not clear which metabolite(s) are responsible for the significant rheological changes occurring in the dough matrix at the initial stage of the fermentation process. Verheyen et al. (2014) equally reported a significant decrease in $G^{*}$ and increase in $\delta$ at a frequency $f=1 \mathrm{~Hz}$ immediately after the mixing of yeast-containing dough. They attributed this effect to the release of glutathione by dead yeast cells in the dough. Whereas the concentration of the other main yeast metabolites (ethanol, succinic acid, glycerol) was found to in- 
crease gradually over time (Jayaram et al., 2013), small amounts of glutathione may indeed be present in dough already at the beginning of fermentation, and may bring about a substantial softening of the dough matrix (Verheyen et al., 2015). However, no experimental evidence could be found that glutathione specifically targets the dough response at low frequencies (Meerts et al., 2018).

Regardless of the exact underlying mechanism, both the time sweep and the frequency sweep seem to suggest that yeast typically affects the dough rheology in two distinct ways, either via the production of $\mathrm{CO}_{2}$ gas (thus lowering the dough density) or via the production of other rheologically active metabolites (which may have a direct impact on the viscoelasticity of the dough matrix). In bread production, salt and sugar are added to achieve a better control of the fermentation process (amongst other purposes). Both of these components have the ability to change the structure of the dough matrix as well as the fermentation kinetics. In the final part of this study, the effect of the salt and sugar concentration on the rheological properties and volumetric expansion profile of fermenting dough is determined with the parallel-plate setup with expanding gap. In addition, a rheofermentometer is used to study the changes in the gas-retaining properties of dough as the salt and sugar concentrations are varied.

\subsection{Case study 1: Impact of salt}

Salt $(\mathrm{NaCl})$ constitutes an essential ingredient in bread. The addition of salt strengthens the dough and stabilises the yeast fermentation rate, hence providing a better control of the fermentation process (Miller and Hoseney, 2008; Beck et al., 2012). Excessive daily intake of salt $(\mathrm{NaCl})$ has been identified as one of the primary causes for elevated blood pressure, which might lead to cardiovascular diseases (Lynch et al., 2009). It is therefore important to understand the impact of salt so that this component can be reduced or (partially) replaced in the dough recipe (Miller and Hoseney, 2008).

\subsubsection{Unfermented dough}

The dough recipe used in this study includes $1.5 \mathrm{wt} \%$ of salt $(\mathrm{NaCl})$, which agrees well with the levels currently used in industry $(1.2 \mathrm{wt} \%-2 \mathrm{wt} \%$, according to Lynch et al. (2009) and McCann and Day (2013)). Fig. 5 shows how the dynamic moduli and the extensional response of unfermented dough are affected by the presence of salt. Salt turns out to have only a limited effect on the dynamic moduli (Fig. 5a). The difference in $G^{\prime}$ and $G^{\prime \prime}$ between the two dough systems is statistically significant only at the lowest frequencies. Other studies reported a small increase (Beck et al., 2012), a slight decrease (Angioloni and Dalla Rosa, 2005; Salvador et al., 2006; Lynch et al., 2009), or no change at all (Wehrle et al., 1997; McCann and Day, 2013) in the dynamic moduli upon addition of salt. The disagreement between the various studies is probably caused by the fact that the gluten response is largely masked by that of the starch in linear oscillatory tests (McCann and Day, 2013; Meerts et al., 2017a). By contrast, non-linear rheological 
tests are known to be very sensitive to changes in the gluten network (Amemiya and Menjivar, 1992). The strengthening effect of salt is clearly visible in the extensional response of the unfermented dough, mainly at large strains (Fig. 5b). The enhanced strain-hardening behaviour can be traced back to the fibril-like structure assumed by the gluten network in the presence of salt, as evidenced by confocal laser scanning microscopy images (Beck et al., 2012; McCann and Day, 2013). The salt ions are believed to shield the charged protein residues of the gluten network, leading to enhanced van der Waals interactions and delayed protein hydration (McCann and Day, 2013). The increased resistance against stretching of salt-supplemented dough was also observed in extensograph (Harinder and Bains, 1990; Lynch et al., 2009) and Kieffer rig tests (Lynch et al., 2009; Beck et al., 2012). Several studies (Lynch et al., 2009; Beck et al., 2012) additionally report an increase in the dough's extensibility upon addition of salt. The decreased slope of $\eta_{e}^{+}(\epsilon)$ at large strains in Fig. 5b seems to suggest indeed that the dough without salt is about to experience failure at $\epsilon=2.9$, whereas for the dough with salt there are no signs yet of imminent failure. It can thus be concluded that extensional tests are well-suited to probe the strengthening effect of salt with regard to the gluten network.

\subsubsection{Fermenting dough}

The addition of salt to wheat flour dough typically results in larger bread volumes (He et al., 1992; McCann and Day, 2013), which might reflect the improved strength of the gluten network, which would indeed result in a better gas-holding capacity. Yet, salt also has an impact on the kinetics of yeast fermentation. The reduction in fermentation rate associated with the addition of salt enables a better control of the fermentation process, and provides the dough with an improved texture (Miller and Hoseney, 2008). The gaseous release curves obtained with the rheofermentometer for fermenting dough without salt and with salt are shown in Fig. 6a and Fig. 6c, respectively. In case salt is included in the dough recipe, the (maximum) $\mathrm{CO}_{2}$ production rate decreases significantly, as does the total amount of $\mathrm{CO}_{2}$ produced within the time span of two hours (from $1203 \pm 27 \mathrm{ml}$ to $987 \pm 36 \mathrm{ml}$ ). The relative amount of gas retained within the dough does not differ significantly for the two conditions tested (being about $93 \%$ ), which implies that the dough without salt actually contains $20 \%$ more gas than the dough with salt. The increase in gluten network strength due to the presence of salt, as evidenced by the extensional tests, thus proves insufficient to improve the dough's gasholding capacity to a significant extent. The resulting dynamic moduli of fermenting dough prepared with or without salt are shown in Fig. 7. The moduli were rescaled with the help of the calibration curve established in section 3.1.3 to account for the overfilling contributions. Changes in the salt concentration (and sugar concentration, see section 3.4) affect to a limited extent the initial volume and consistency of the dough sample at the loading step, thus giving rise to slightly different values for the initial gap $H_{0}$ for each condition. The scale factors provided by the calibration curve depend on both $H / D$ and the overfilling volume, yet the differences in these two characteristics for the different conditions were found to be so small that they could safely be ignored. 
Hence, for all salt and sugar concentrations, the same calibration curve was used to account for the effect of overfilling. In Fig. 7, the rescaled dynamic moduli have also been normalised using their initial values at time $t=0$ in order to present the relative changes more clearly (the difference in the initial values being limited to approx. $7 \%$ ). With salt, the drop in the dynamic moduli occurs later and is also less pronounced, which is in line with the gas production data obtained with the rheofermentometer. The time evolution of $G^{\prime}(t)$ can be captured fairly well by a single exponential decay function:

$$
\frac{G^{\prime}(t)-G_{\mathrm{e}}^{\prime}}{G_{0}^{\prime}-G_{\mathrm{e}}^{\prime}}=\exp \left(-t / t_{\mathrm{d}}\right)
$$

In this expression $G_{0}^{\prime}[\mathrm{Pa}]$ represents the initial value of $G^{\prime}(t)$ at $t=0$ and $G_{\mathrm{e}}^{\prime}[\mathrm{Pa}]$ its steady-state value. The parameter $t_{\mathrm{d}}[\mathrm{s}]$ is the time constant of the exponential decay function. In Fig. $7 G^{\prime}(t)$ is expressed relative to its initial value $G_{0}^{\prime}$, in which case the exponential decay model can be rewritten as:

$$
\frac{G^{\prime}(t)}{G_{0}^{\prime}}=\frac{G_{\mathrm{e}}^{\prime}}{G_{0}^{\prime}}+\frac{G_{0}^{\prime}-G_{\mathrm{e}}^{\prime}}{G_{0}^{\prime}} \exp \left(-t / t_{\mathrm{d}}\right)
$$

Upon the addition of salt, the value of the time constant $t_{\mathrm{d}}$ increases, and so does the ratio of $G_{\mathrm{e}}^{\prime}$ compared to $G_{0}^{\prime}$ (see Table 1). The sigmoidal expansion profile of fermenting dough can be described accurately with a modified Gompertz model, as proposed by Romano et al. (2007):

$$
\frac{V(t)}{V_{0}}=1+b_{1} \cdot \exp \left(-\exp \left(\frac{b_{2} e}{b_{1}}\left(t_{\mathrm{lag}}-t\right)+1\right)\right)
$$

The parameters of this model can be related directly to the characteristics of the leavening process: the parameter $b_{1}[-]$ represents the maximum volume expansion ratio $\left(=\frac{V_{e}-V_{0}}{V_{0}}\right)$ of the fermenting dough sample; $b_{2}\left[\mathrm{~s}^{-1}\right]$ corresponds to the maximum specific volume growth rate; and finally $t_{\text {lag }}[\mathrm{s}]$ stands for the time lag of the leavening process. The symbol $e$ represents Euler's number. Despite the difference in the amount of gas being produced in the rheofermentometer, the maximum relative volume reached by the fermenting dough in the rheometer is fairly similar for both cases (maximum $V_{e} / V_{0}=4.4$, see inset Fig. 7). There is, however, a clear difference in the rate of volume expansion, as indicated by the value of the parameter $t_{\text {lag }}$ in Table 1 . Moreover, at the very end of the two-hour fermentation period the volume of the fermenting dough without salt suddenly decreases again to $V_{e} / V_{0}=4.26$, whereas that of the salt-containing dough remains stable, thus indicating a strengthening effect of salt, which is in line with the extensional rheology data. In another series of rheofermentometer tests performed by Lynch et al. (2009) the stabilising effect of salt is more noticeable. Lynch et al. (2009) found that while the presence of salt indeed reduces fermentation activity, and thus gas production, the gluten network is also strengthened and consequently the relative amount of gas retained within the dough increases significantly. 


\subsection{Case study 2: Impact of sugar}

A substantial amount of sucrose (6 wt\% or more) is often included in the dough recipe as a supplementary nutrient source for the yeast cells and to improve bread flavour (Myers et al., 1998; Struyf et al., 2017). To investigate the impact of changes in the sugar content on the rheological behaviour of dough and the fermentation process, doughs were prepared with or without additional sucrose and subsequently tested both in the unfermented and the fermenting state.

\subsubsection{Unfermented dough}

Including sucrose $(6 \mathrm{wt} \%)$ in the dough recipe has a clear impact on the rheological behaviour of unfermented dough (Fig. 5). In case sucrose is added, the dynamic moduli are shifted downwards, yet their frequency dependence is not really affected (Fig. 5a). Similar trends were observed by Salvador et al. (2006). Fig. 5b shows that the extensional viscosity decreases as well, but predominantly for the small strains. These changes in rheological behaviour resemble very closely the rheological impact of an increase in the amount of water used in the dough recipe, which we have studied in a previous publication (Meerts et al., 2017b). When preparing the dough, sucrose is added as an aqueous solution to the wheat flour, while keeping the total amount of water used in the dough preparation the same for all dough samples. However, the dissolution of sucrose in water leads to a small volume expansion. Consequently, the volume fraction of the mobile phase present in dough increases slightly, and the dough behaviour is affected in a way similar to that of dough prepared with additional water. Linear oscillatory tests on dough containing $0.99 \mathrm{ml}$ sucrose solution or $0.99 \mathrm{ml}$ water yielded no significant differences (results not shown), indicating that the observed weakening effect of adding sucrose can indeed be explained by a change in volume of the mobile phase. Salvador et al. (2006) attributed the lower dynamic moduli in sugar-containing dough to an insufficient buildup of the gluten network as the gluten proteins and sucrose both compete for water. However, they equally mention that sucrose might not have sufficient hydroxyl groups available for hydrogen bonding with water to create such a competition. Moreover, an insufficient buildup of the gluten network would be readily revealed in the strain-hardening behaviour of dough (as is the case for under- or overmixed dough, see Meerts et al. (2017b)), but as Fig. 5b indicates, the dough prepared with sucrose still exhibits a substantial degree of strain-hardening. Changes in the water content do not impair the strain-hardening properties of dough (Meerts et al., 2017b), and so the results obtained in this study seem to point mainly to a volume effect for sucrose. 


\subsubsection{Fermenting dough}

The impact of changing the amount of sucrose available to the yeast on the gas production is shown in Figs. 6b and 6c. When additional sucrose is included in the dough recipe, the yeast exhibits higher fermentation activity. The total amount of $\mathrm{CO}_{2}$ produced over the course of two hours increases considerably (from $783 \pm 13 \mathrm{ml}$ to 987 $\pm 36 \mathrm{ml}$ ), to such an extent that the sugar-containing dough is no longer capable of retaining all the gas produced. Whereas a substantial fraction of the produced $\mathrm{CO}_{2}$ is thus released to the atmosphere, the high-sugar dough still contains significantly more $\mathrm{CO}_{2}$ gas as compared to the low-sugar dough. Fig. 7 gives the dynamic moduli of the fermenting dough samples with or without additional sucrose. At the early stages of the fermentation process the drop in the dynamic moduli is somewhat less pronounced for the high-sugar dough as compared to the low-sugar dough, as evidenced by the highsugar dough having a higher value for the parameter $t_{\mathrm{d}}$ in Table 1 . In a similar fashion, the gaseous release curves (Fig. 6) indicate that at the start of fermentation, the yeast produces less gas when more sucrose is available. This counter-intuitive effect results from the osmotic stress induced in yeast by sucrose when present in high amounts (Myers et al., 1998). However, without additional sucrose the yeast quickly runs out of readily fermentable sugars, and is forced to switch to the fermentation of maltose. During this readjustment, the gas production is likely to decline (Loveday and Winger, 2007; Struyf et al., 2017). In addition, during fermentation yeast produces small amounts of glycerol, which tend to accumulate inside the yeast cells and so protect them against the low extracellular water activity created by sugar and/or salt (Nevoigt and Stahl, 1997). Consequently, when seen over the course of two hours the total amount of gas produced is still higher in case the yeast is supplemented with additional sucrose. The inset in Fig. 7 indicates that the high-sugar dough indeed appears to expand slightly more as compared to the low-sugar dough, yet this small difference is not statistically significant. Table 1 also indicates that the high-sugar dough exhibits slightly higher values for the maximum expansion ratio $b_{1}$ and the time lag $t_{\text {lag }}$ than the low-sugar dough. Finally, at the end of the fermentation period, the relative decline in the dynamic moduli is also somewhat more pronounced for the high-sugar dough, yet again differences are not statistically significant.

\section{Conclusions}

The use of a parallel-plate setup with adjustable gap and overflow sample cup allows to track the changes in the linear viscoelastic behaviour of complex biological materials with changing volume such as fermenting dough in-situ and time-resolved. Despite the intrinsic fragility of fermenting dough samples, the rheological response turns out to be fairly robust, as different normal force protocols yield very comparable results, and good reproducibility is obtained throughout. The effect of overfilling can be taken into account by establishing a calibration curve; however, for fermenting dough this curve is not straightforward to obtain as without the support of the sample edges, the dough 
samples are prone to collapse. After calibration, information on both the dynamic moduli and the sample density can be acquired in a single rheological experiment. In the case of fermenting dough, the dynamic moduli and the sample density did not follow exactly the same trend, as besides $\mathrm{CO}_{2}$ yeast produces several other metabolites, which may affect the rheology of the dough matrix as well. The combined use of dynamic measurements with a rheofermentometer proved to be a valuable approach to trace the impact of sugar and salt on both the rheology of the dough matrix as well as the intensity and extent of the fermentation process.

\section{Acknowledgments}

MM is indebted to the Research Foundation - Flanders (FWO) for a doctoral fellowship at KU Leuven. The authors would also like to express their gratitude to the Research Fund KU Leuven (IDO/12/011) for financial support. Nore Struyf and Mohammad Naser Rezaei are gratefully acknowledged for determining the flour characteristics (protein content, moisture content, optimal mixing time and water absorption).

\section{References}

AACC International, Approved Methods of Analysis (11th edition), AACC International (2000), St. Paul (MN).

Amemiya, J. I., Menjivar, J. A., 1992. Comparison of small and large deformation measurements to characterize the rheology of wheat flour doughs. Journal of Food Engineering 16, 91-108.

AOAC International, Official Methods of Analysis of AOAC international (16th edition), AOAC (1995), Washington, DC.

Angioloni, A., Dalla Rosa, M., 2005. Dough thermo-mechanical properties: influence of sodium chloride, mixing time and equipment. Journal of Cereal Science 41, 327-331.

Aslankoohi, E., Rezaei, M.N., Vervoort, Y., Courtin, C.M., Verstrepen, K.J., 2015. Glycerol production by fermenting yeast cells is essential for optimal bread dough fermentation. PLoS ONE 10:e0119364.

Baker, J.C., Mize, M.D., 1941. The origin of the gas cell in bread dough. Cereal Chemistry $18,19-34$.

Beck, M., Jekle, M., Becker, T., 2012. Impact of sodium chloride on wheat flour dough for yeast-leavened products. I. Rheological attributes. Journal of the Science of Food and Agriculture 92, 585-592.

Cauvain, S.P., Young, L.S, Technology of Breadmaking (2nd edition), Springer (2007), New York (NY). 
Chevallier, S., Zúñiga, R., Le-Bail, A., 2012. Assessment of bread dough expansion during fermentation. Food and Bioprocess Technology 5, 609-617.

Czuchajowska, Z., Pomeranz, Y., 1993. Gas formation and gas retention. I. The system and methodology. Cereal Foods World 38, 499-503.

Elmehdi, H.M., Page, J.H., Scanlon, M.G., 2003. Monitoring dough fermentation using acoustic waves. Food and Bioproducts Processing 81, 217-223.

Elmehdi, H.M., Page, J.H., Scanlon, M.G., 2007. Evaluating dough density changes during fermentation by different techniques. Cereal Chemistry 84, 250-252.

Ewoldt, R.H., Johnston, M.T., Caretta, L.M., Experimental challenges of shear rheology: how to avoid bad data. In: Complex Fluids in Biological Systems (edited by Spagnolie, S.E.), Springer Science+Business Media (2015), New York (NY).

Harinder, K., Bains, G.S., 1990. High $\alpha$-amylase flours: effect of pH, acid, and salt on the rheological properties of dough. Cereal Chemistry 67, 588-594.

He, H., Roach, R.R., Hoseney, R.C., 1992. Effect of nonchaotropic salts on flour breadmaking properties. Cereal Chemistry 69, 366-371.

Hoseney, R.C., Hsu, K.H., Junge, R.C., 1979. A simple spread test to measure the rheological properties of fermenting dough. Cereal Chemistry 56, 141-143.

Ito, M., Yoshikawa, S., Asami, K., Hanai, T., 1992. Dielectric monitoring of gas production in fermenting bread dough. Cereal Chemistry 69, 325-327.

Jayaram, V.B., Cuyvers, S., Lagrain, B., Verstrepen, K.J., Delcour, J.A., Courtin, C.M., 2013. Mapping of Saccharomyces cerevisiae metabolites in fermenting wheat straightdough reveals succinic acid as pH-determining factor. Food Chemistry 136, 301-308.

Jayaram, V.B., Rezaei, M.N., Cuyvers, S., Verstrepen, K.J., Delcour, J.A., Courtin, C.M., 2014a. Ethanol at levels produced by Saccharomyces cerevisiae during wheat dough fermentation has a strong impact on dough properties. Journal of Agricultural and Food Chemistry 62, 9326-9335.

Jayaram, V.B., Cuyvers, S., Verstrepen, K.J., Delcour, J.A., Courtin, C.M., 2014b. Succinic acid in levels produced by yeast (Saccharomyces cerevisiae) during fermentation strongly impacts wheat bread dough properties. Food Chemistry 151, 421-428.

Kilborn, R.H., Preston, K.R., 1982. A modified extensigraph procedure for measuring the stretching properties of fermented dough. Cereal Chemistry 59, 381-384.

Koksel, F., Scanlon, M.G., Page, J.H., 2016. Ultrasound as a tool to study bubbles in dough and dough mechanical properties: a review. Food Research International 89, 74-89. 
Lee, S., Pyrak-Nolte, L.J., Campanella, O., 2004. Determination of ultrasonic-based rheological properties of dough during fermentation. Journal of Texture Studies 35, 33-52.

Lee, S., Campanella, O., 2013. Impulse viscoelastic characterization of wheat flour dough during fermentation. Journal of Food Engineering 118, 266-270.

Loveday, S.M., Winger, R.J., 2007. Mathematical model of sugar uptake in fermenting yeasted dough. Journal of Agricultural and Food Chemistry 55, 6325-6329.

Lynch, E.J., Dal Bello, F., Sheehan, E.M., Cashman, K.D., Arendt, E.K., 2009. Fundamental studies on the reduction of salt on dough and bread characteristics. Food Research International 42, 885-891.

McCann, T.H., Day, L., 2013. Effect of sodium chloride on gluten network formation, dough microstructure and rheology in relation to breadmaking. Journal of Cereal Science 57, 444-452.

McNaught, A.D., Wilkinson, A., Compendium of Chemical Terminology (2nd edition), Blackwell Scientific Publications (1997), Oxford (UK).

Meerts, M., Cardinaels, R., Oosterlinck, F., Courtin, C.M., Moldenaers, P., 2017a. The interplay between the main flour constituents in the rheological behaviour of wheat flour dough. Food and Bioprocess Technology 10, 249-265.

Meerts, M., Cardinaels, R., Oosterlinck, F., Courtin, C.M., Moldenaers, P., 2017b. The impact of water content and mixing time on the linear and non-linear rheology of wheat flour dough. Food Biophysics 12, 151-163.

Meerts, M., Ramirez Cervera, A., Struyf, N., Cardinaels, R., Courtin, C.M., Moldenaers, P., 2018. The effects of yeast metabolites on the rheological behaviour of the dough matrix in fermented wheat flour dough. Journal of Cereal Science 82, 183-189.

Mert, B., 2008. A new instrumental setup for determination of small amplitude viscoelastic properties of dough during fermentation. European Food Research and Technology $227,151-157$.

Miller, R.A., Hoseney, R.C., 2008. Role of salt in baking. Cereal Foods World 53, 4-6.

Mora, E., Artavia, L.D., Macosko, C.W., 1991. Modulus development during reactive urethane foaming. Journal of Rheology 35, 921-940.

Myers, D.K., Joseph, V.M., Pehm, S., Galvagno, M., Attfield, P.V., 1998. Loading of Saccharomyces cerevisiae with glycerol leads to enhanced fermentation in sweet bread doughs. Food Microbiology 15, 51-58.

Neff, R.A., Macosko, C.W., 1996. Simultaneous measurement of viscoelastic changes and cell opening during processing of flexible polyurethane foam. Rheologica Acta 35, 656-666. 
Nevoigt, E., Stahl, U., 1997. Osmoregulation and glycerol metabolism in the yeast Saccharomyces cerevisiae. FEMS Microbiology Reviews 21, 231-241.

Romano, A., Toraldo, G., Cavella, S., Masi, P., 2007. Description of leavening of bread dough with mathematical modelling. Journal of Food Engineering 83, 142-148.

Salvador, A., Sanz, T., Fiszman, S.M., 2006. Dynamic rheological characteristics of wheat flour-water doughs. Effect of adding $\mathrm{NaCl}$, sucrose and yeast. Food Hydrocolloids 20, 780-786.

Scanlon, M.G., Zghal, M.C., 2001. Bread properties and crumb structure. Food Research International 34, 841-864.

Skaf, A., Nassar, G., Lefebvre, F., Nongaillard, B., 2009. A new acoustic technique to monitor bread dough during the fermentation phase. Journal of Food Engineering 93, 365-378.

Struyf, N., Van der Maelen, E., Hemdane, S., Verspreet, J., Verstrepen, K.J., Courtin, C.M., 2017. Bread dough and baker's yeast: an uplifting synergy. Comprehensive Reviews in Food Science and Food Safety 16, 850-867.

Verheyen, C., Jekle, M., Becker, T., 2014. Effects of Saccharomyces cerevisiae on the structural kinetics of wheat dough during fermentation. LWT - Food Science and Technology 58, 194-202.

Verheyen, C., Albrecht, A., Herrmann, J., Strobl, M., Jekle, M., Becker, T., 2015. The contribution of glutathione to the destabilizing effect of yeast on wheat dough. Food Chemistry 173, 243-249.

Wehrle, K., Grau, H., Arendt, E.K., 1997. Effects of lactic acid, acetic acid, and table salt on fundamental rheological properties of wheat dough. Cereal Chemistry 74, 739744 . 


\section{List of Figures}

1 (a) Schematic drawing of the rheological setup used for the study of fermenting dough. Dimensions can be found in the text. (b) External view of the rheological setup. (c) View of the dough fermenting inside the rheological setup. The PMMA support rings were removed to allow an internal view of the setup. . . . . . . . . . . . . . .

2 The evolution of the storage modulus $G^{\prime}(t)$ (squares), loss modulus $G^{\prime \prime}(t)$ (diamonds), phase angle $\delta(t)$ (circles) (a) and of the measurement gap $H(t)$ (circles) (b) for fermenting dough over the course of two hours. The normal force $F_{n}(t)$ (triangles) (b) was varied linearly from $0.5 \mathrm{~N}$ to $0.05 \mathrm{~N}$ during the first $30 \mathrm{~min}$, and subsequently held constant at 0.05 $\mathrm{N}$. The dynamic moduli were obtained at $\omega=1 \mathrm{rad} / \mathrm{s}$ and $\gamma_{0}=0.06 \%$. Measurements were done on three different samples prepared on different days (as shown by the different colours), and indicate good reproducibility. 26

3 (a) The dynamic moduli $G^{\prime}(t)$ (squares) and $G^{\prime \prime}(t)$ (diamonds) for fermenting dough (black) and their rescaled counterparts (white), taking into account the effect of overfilling. Inset: the calibration curve for the dynamic moduli obtained for unfermented dough based on five $(H, D)$ data points relevant for fermenting dough. (b) The relative increase in measurement gap $H(t)$ and sample volume $V(t)$ for fermenting dough, with the corresponding initial values taken as the reference. Inset: the relative increase in sample diameter $D(t) / D_{0}$ as a function of the relative increase in measurement gap $H(t) / H_{0}$. The knowledge of both $H(t) / H_{0}$ and $D(t) / D_{0}$ allows to determine $V(t) / V_{0}$. Error bars indicate the standard deviation. Note: the interruptions in each of the curves correspond to the time intervals during which frequency sweep data were acquired in multiwave mode. . . . . . . . . . . . . . . . . . . . . .

4 (a) The time evolution of the rescaled dynamic moduli $G^{\prime}(t)$ (squares) and $G^{\prime \prime}(t)$ (diamonds) and of the dough density $\rho(t)$ (circles), after normalisation with respect to their initial values $G_{0}^{\prime}, G_{0}^{\prime \prime}$ and $\rho_{0}$. (b) The rescaled dynamic moduli $G^{\prime}(\omega)$ (squares) and $G^{\prime \prime}(\omega)$ (diamonds) and the phase angle $\delta(\omega)$ (circles) plotted versus angular frequency $\omega$ for unfermented dough directly after mixing (black) and for fermenting dough after 15 min (grey) and 90 min of fermentation (white). Measurements were performed in triplicate, and the error bars indicate the standard deviation. For the sake of clarity, error bars are only shown for the final data points in (a) . . . . . . . . . . . . . . . . . . .

5 (a) The dynamic moduli $G^{\prime}(\omega)$ (squares) and $G^{\prime \prime}(\omega)$ (diamonds) and (b) the transient extensional viscosity $\eta_{e}^{+}(\epsilon)$ (circles) for unfermented dough prepared with $6 \mathrm{wt} \%$ sugar and no salt (white); no sugar and $1.5 \mathrm{wt} \%$ salt (grey); 6 wt\% sugar and $1.5 \mathrm{wt} \%$ salt (black). The dough samples were rested for 6 hours at $32{ }^{\circ} \mathrm{C}$ and at a relative humidity of $75 \%$ prior to testing. Error bars represent the standard deviation. . . . . . . . 
6 The gaseous release curves for fermenting dough prepared with (a) 6 wt\% sugar, no salt; (b) no sugar and $1.5 \mathrm{wt} \%$ salt; (c) 6 wt\% sugar, 1.5 wt\% salt. The area shaded in dark grey represents the amount of $\mathrm{CO}_{2}$ retained within the dough, whereas the area shaded in light grey stands for the amount of $\mathrm{CO}_{2}$ released by the dough. The time $t_{m}$ denotes the instant at which the maximum fermentation rate $F_{m}$ is reached, whereas $t_{x}$ represents the instant at which the dough starts to release gas. . . . .

7 The decrease in the rescaled dynamic moduli $G^{\prime}(t)$ (squares) and $G^{\prime \prime}(t)$ (diamonds) after normalisation with respect to their initial values $G_{0}^{\prime}$ and $G_{0}^{\prime \prime}$ for fermenting dough prepared with $6 \mathrm{wt} \%$ sugar and no salt (white); no sugar and $1.5 \mathrm{wt} \%$ salt (grey); $6 \mathrm{wt} \%$ sugar and $1.5 \mathrm{wt} \%$ salt (black). Inset: the corresponding increase in volume $V(t)$ after normalisation with respect to the initial volume $V_{0}$ for the three conditions. The time evolution of $G^{\prime}(t) / G_{0}^{\prime}$ and $V(t) / V_{0}$ can be described by an exponential decay function (eq. 9) and by the modified Gompertz model (eq. 10), respectively, as indicated by the black lines. All measurements were performed in triplicate. For the sake of clarity, error bars indicating the standard deviation are only shown for the final data points. . . . . . . . . . . . . . 31 


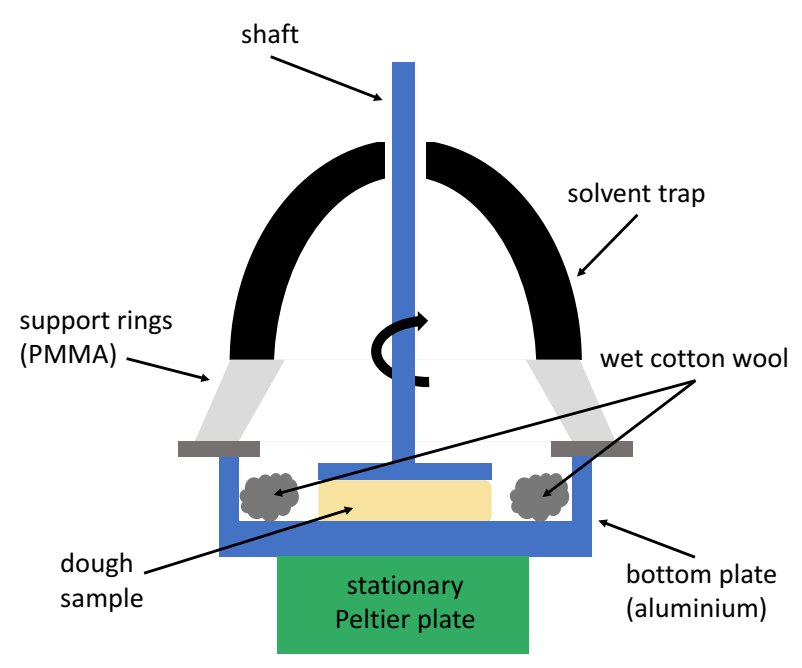

(a)

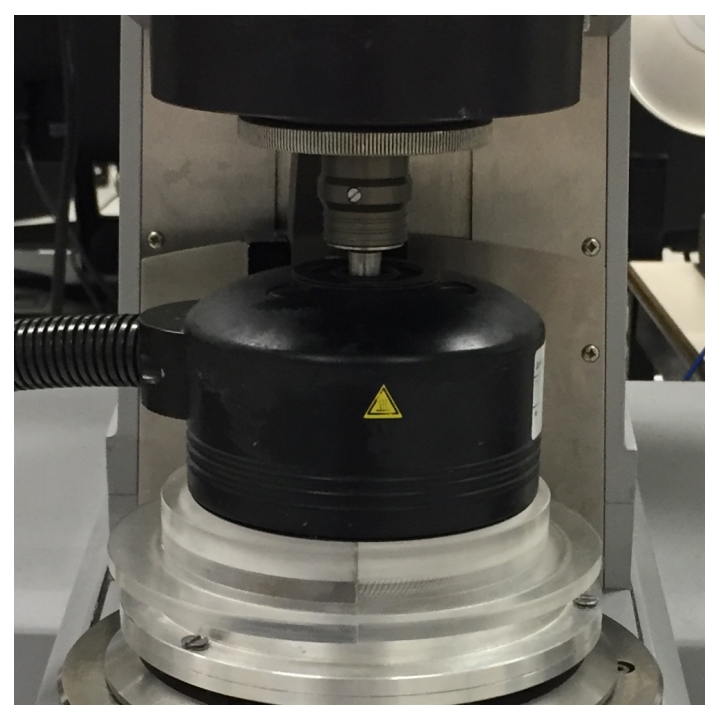

(b)

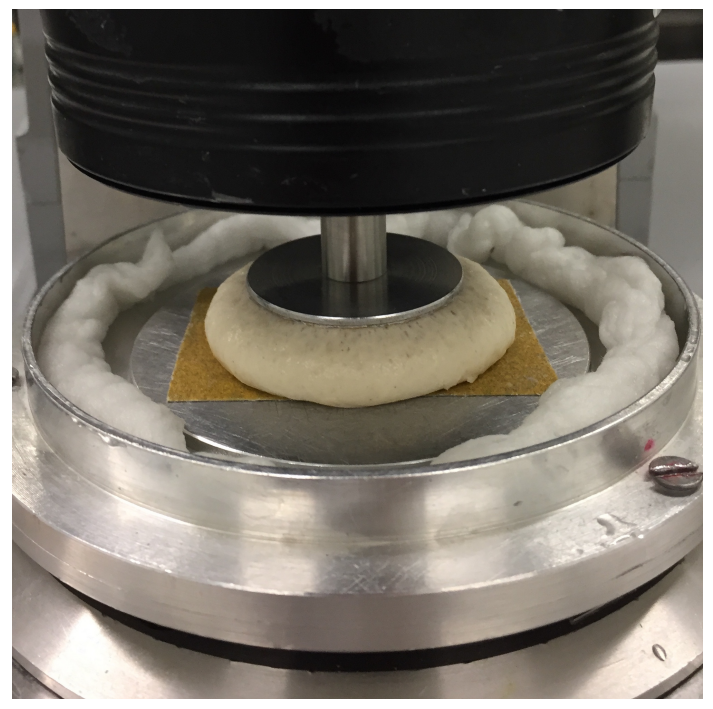

(c)

Figure 1: (a) Schematic drawing of the rheological setup used for the study of fermenting dough. Dimensions can be found in the text. (b) External view of the rheological setup. (c) View of the dough fermenting inside the rheological setup. The PMMA support rings were removed to allow an internal view of the setup. 


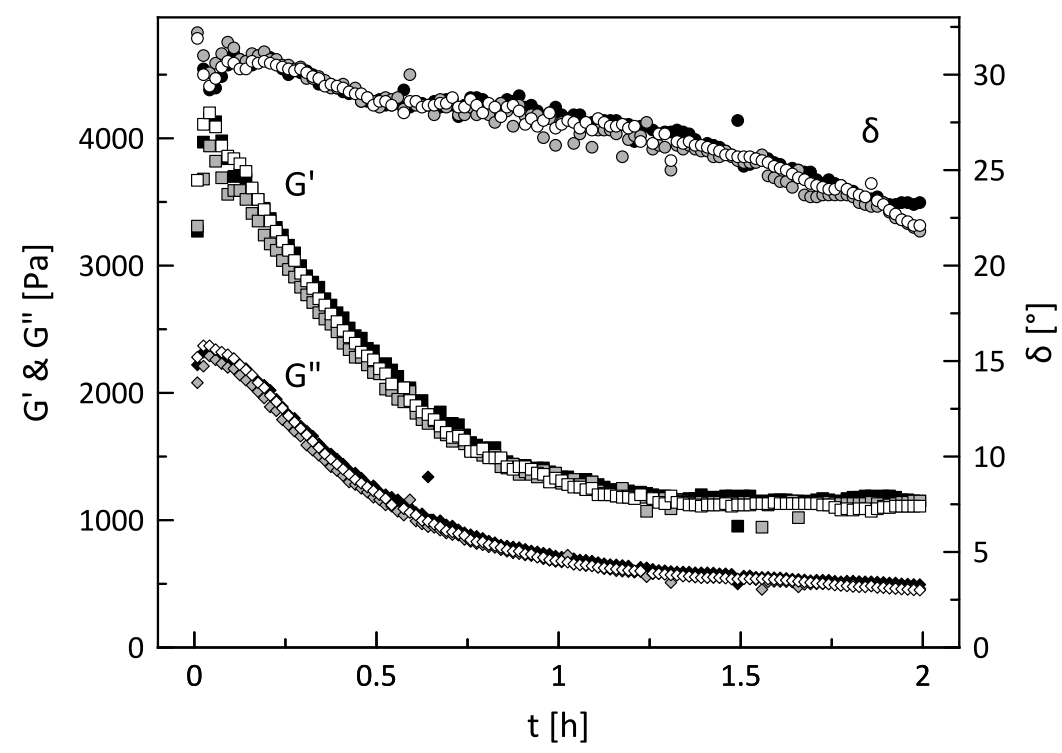

(a)

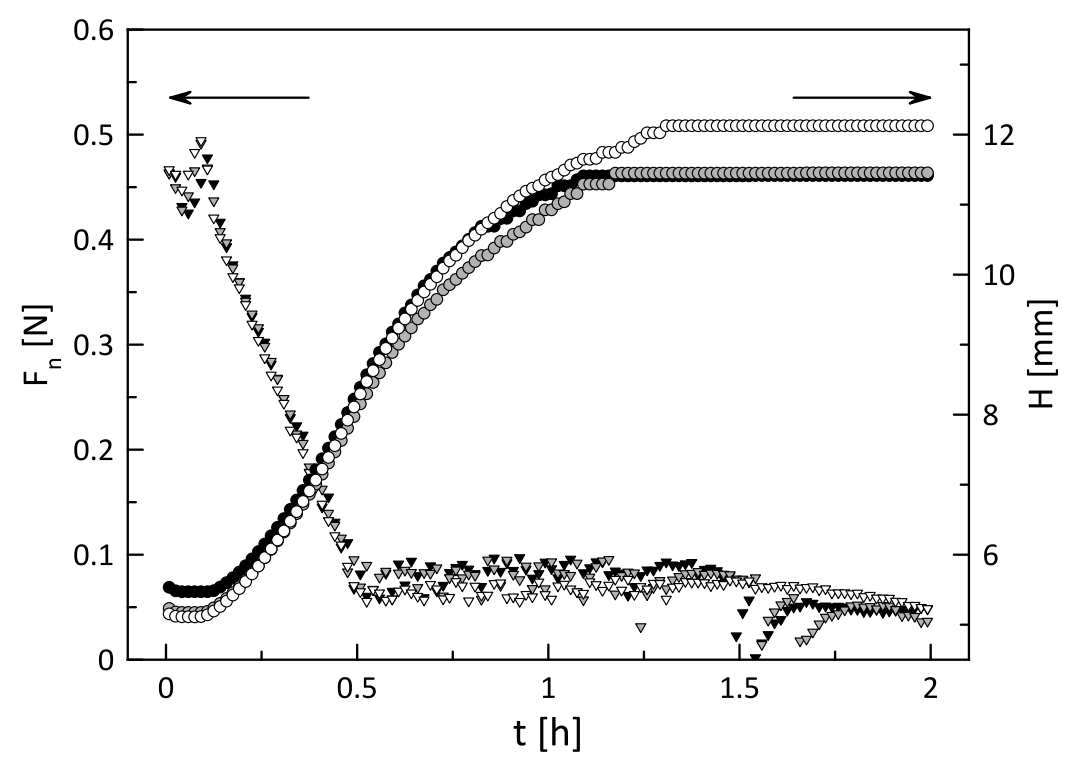

(b)

Figure 2: The evolution of the storage modulus $G^{\prime}(t)$ (squares), loss modulus $G^{\prime \prime}(t)$ (diamonds), phase angle $\delta(t)$ (circles) (a) and of the measurement gap $H(t)$ (circles) (b) for fermenting dough over the course of two hours. The normal force $F_{n}(t)$ (triangles) (b) was varied linearly from $0.5 \mathrm{~N}$ to $0.05 \mathrm{~N}$ during the first $30 \mathrm{~min}$, and subsequently held constant at $0.05 \mathrm{~N}$. The dynamic moduli were obtained at $\omega=1 \mathrm{rad} / \mathrm{s}$ and $\gamma_{0}=$ $0.06 \%$. Measurements were done on three different samples prepared on different days (as shown by the different colours), and indicate good reproducibility. 


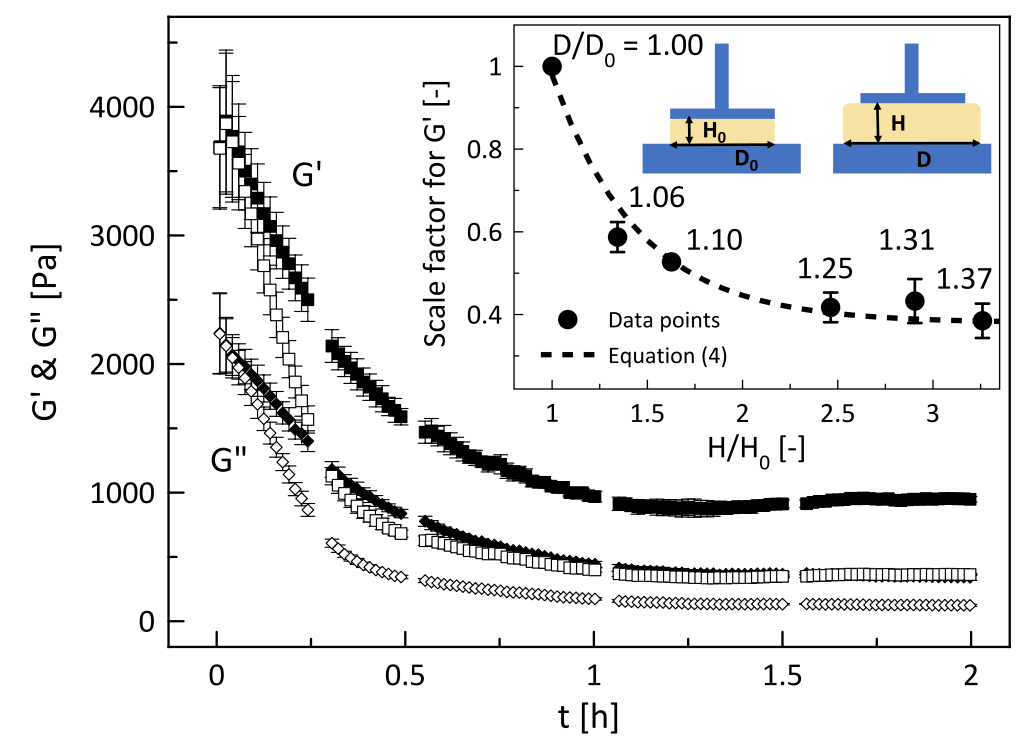

(a)

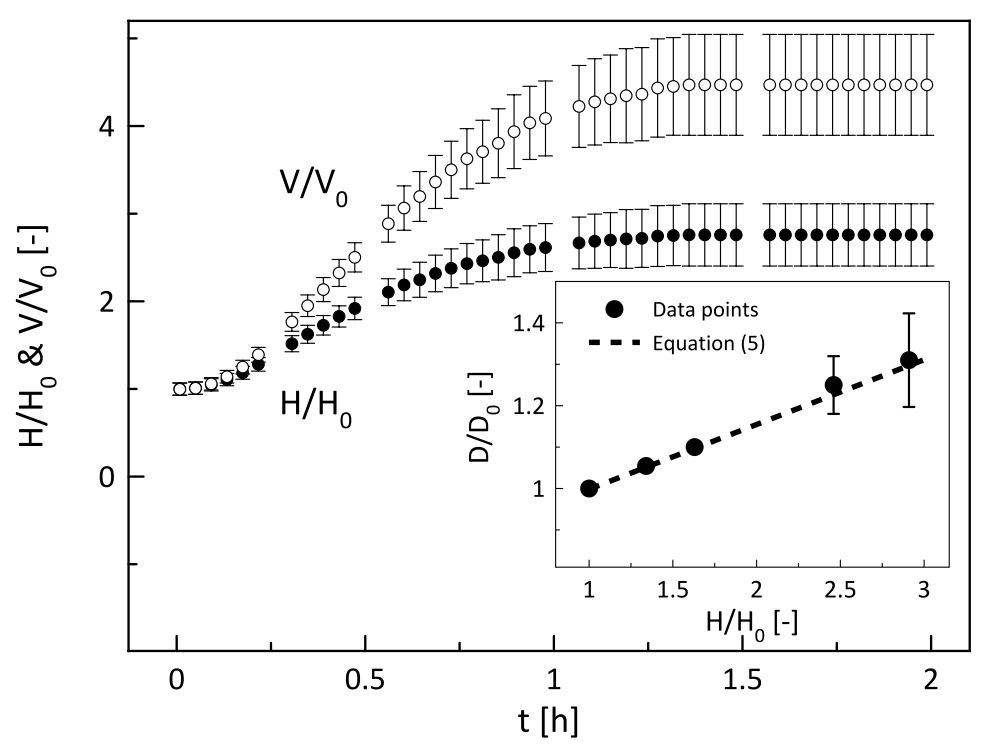

(b)

Figure 3: (a) The dynamic moduli $G^{\prime}(t)$ (squares) and $G^{\prime \prime}(t)$ (diamonds) for fermenting dough (black) and their rescaled counterparts (white), taking into account the effect of overfilling. Inset: the calibration curve for the dynamic moduli obtained for unfermented dough based on five $(H, D)$ data points relevant for fermenting dough. (b) The relative increase in measurement gap $H(t)$ and sample volume $V(t)$ for fermenting dough, with the corresponding initial values taken as the reference. Inset: the relative increase in sample diameter $D(t) / D_{0}$ as a function of the relative increase in measurement gap $H(t) / H_{0}$. The knowledge of both $H(t) / H_{0}$ and $D(t) / D_{0}$ allows to determine $V(t) / V_{0}$. Error bars indicate the standard deviation. Note: the interruptions in each of the curves correspond to the time intervals during which frequency sweep data were acquired in multiwave mode. 


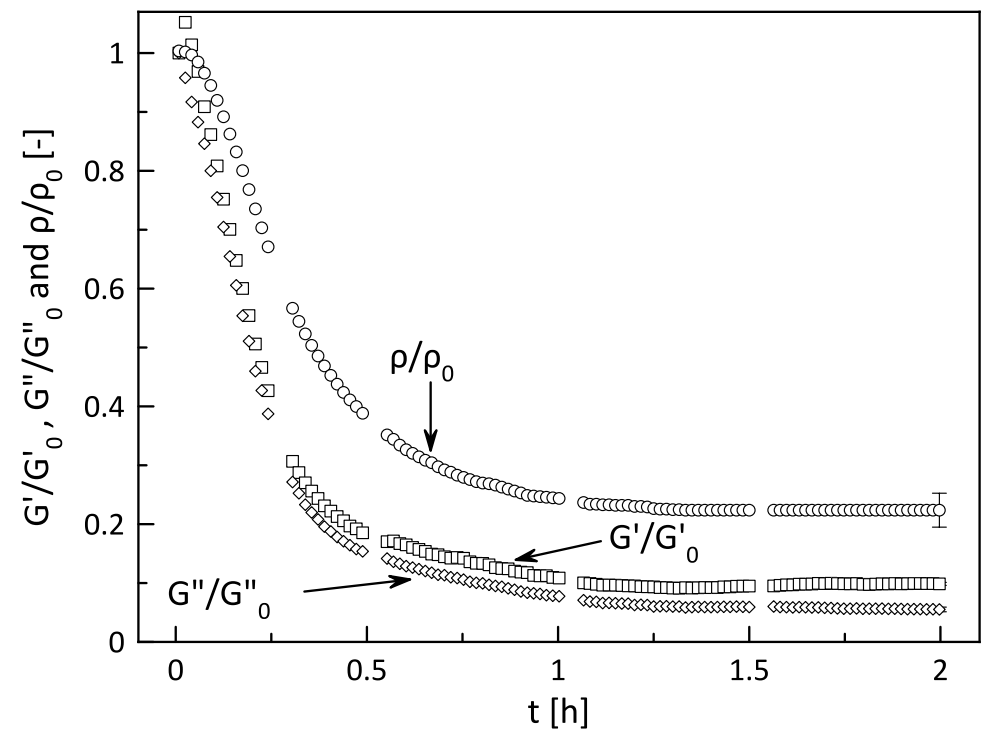

(a)

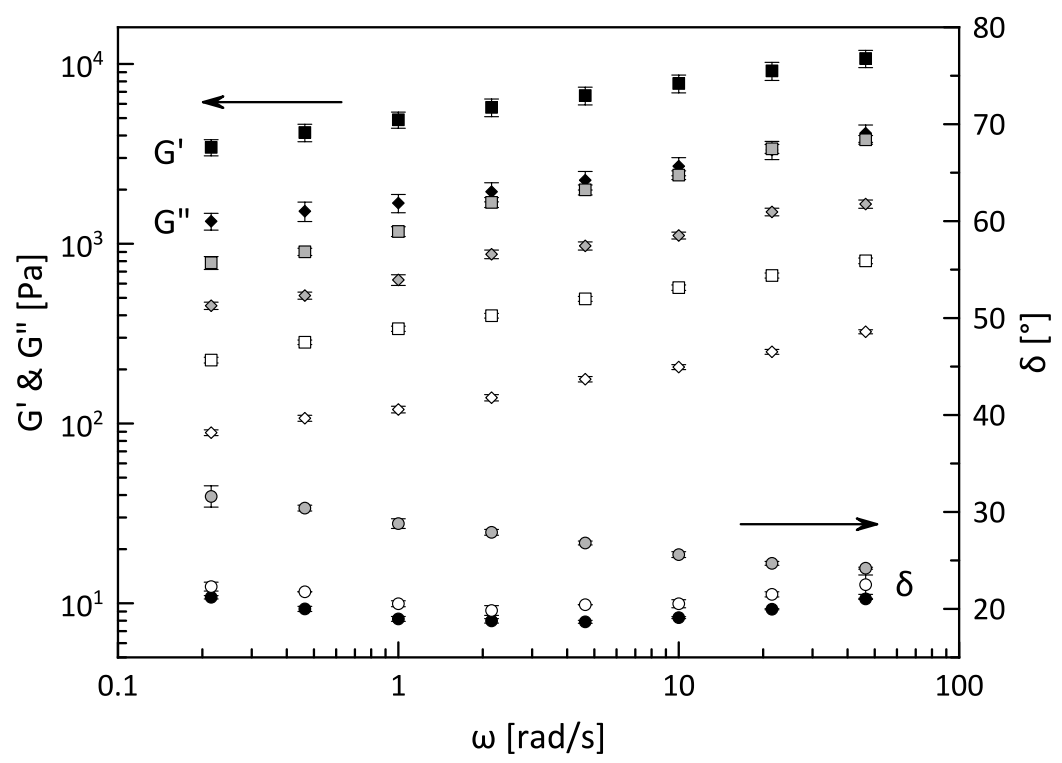

(b)

Figure 4: (a) The time evolution of the rescaled dynamic moduli $G^{\prime}(t)$ (squares) and $G^{\prime \prime}(t)$ (diamonds) and of the dough density $\rho(t)$ (circles), after normalisation with respect to their initial values $G_{0}^{\prime}, G_{0}^{\prime \prime}$ and $\rho_{0}$. (b) The rescaled dynamic moduli $G^{\prime}(\omega)$ (squares) and $G^{\prime \prime}(\omega)$ (diamonds) and the phase angle $\delta(\omega)$ (circles) plotted versus angular frequency $\omega$ for unfermented dough directly after mixing (black) and for fermenting dough after 15 min (grey) and 90 min of fermentation (white). Measurements were performed in triplicate, and the error bars indicate the standard deviation. For the sake of clarity, error bars are only shown for the final data points in (a). 


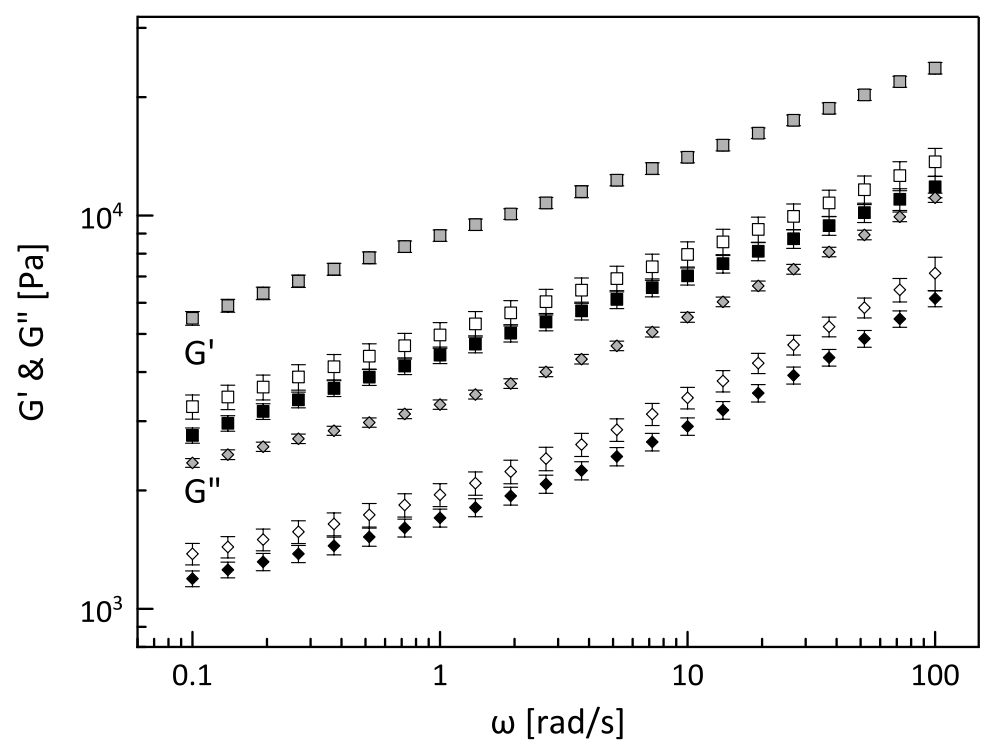

(a)

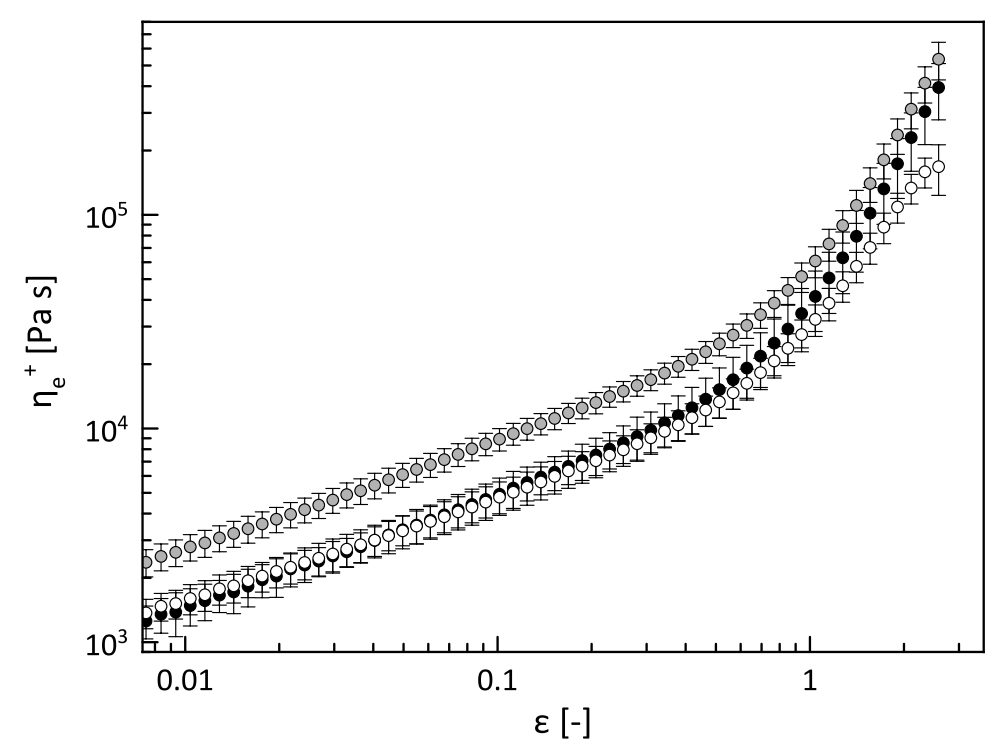

(b)

Figure 5: (a) The dynamic moduli $G^{\prime}(\omega)$ (squares) and $G^{\prime \prime}(\omega)$ (diamonds) and (b) the transient extensional viscosity $\eta_{e}^{+}(\epsilon)$ (circles) for unfermented dough prepared with 6 $\mathrm{wt} \%$ sugar and no salt (white); no sugar and $1.5 \mathrm{wt} \%$ salt (grey); $6 \mathrm{wt} \%$ sugar and 1.5 wt\% salt (black). The dough samples were rested for 6 hours at $32{ }^{\circ} \mathrm{C}$ and at a relative humidity of $75 \%$ prior to testing. Error bars represent the standard deviation. 


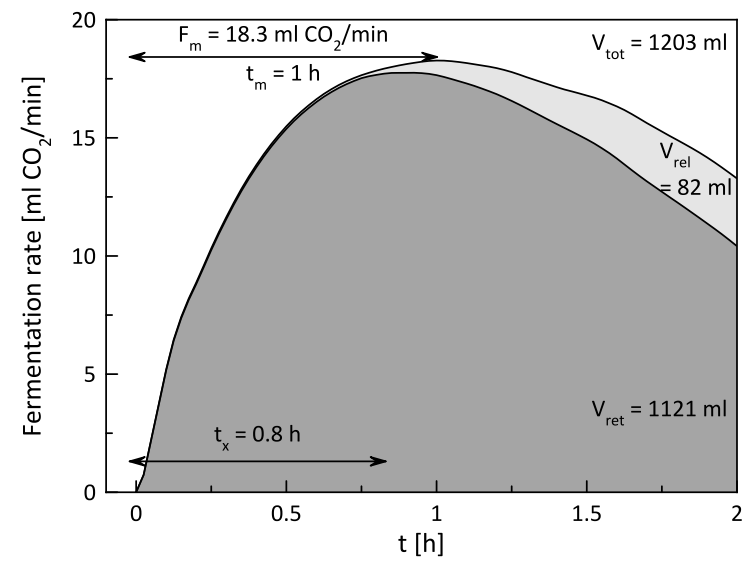

(a)

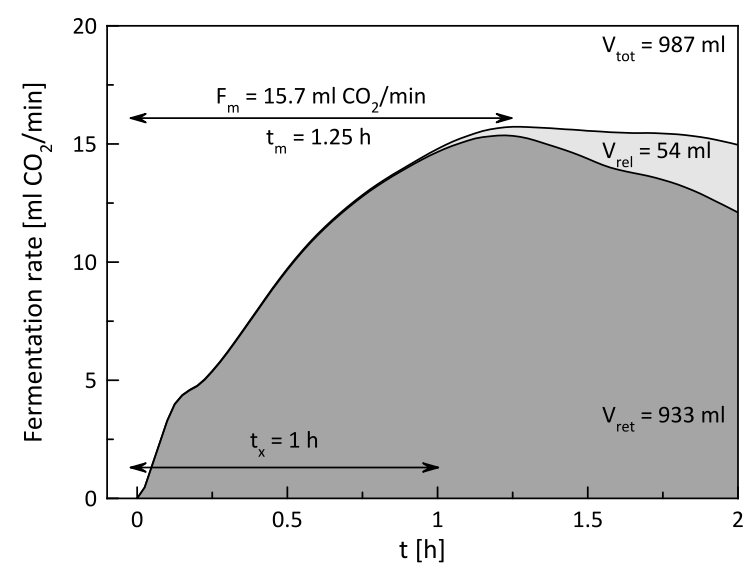

(c)

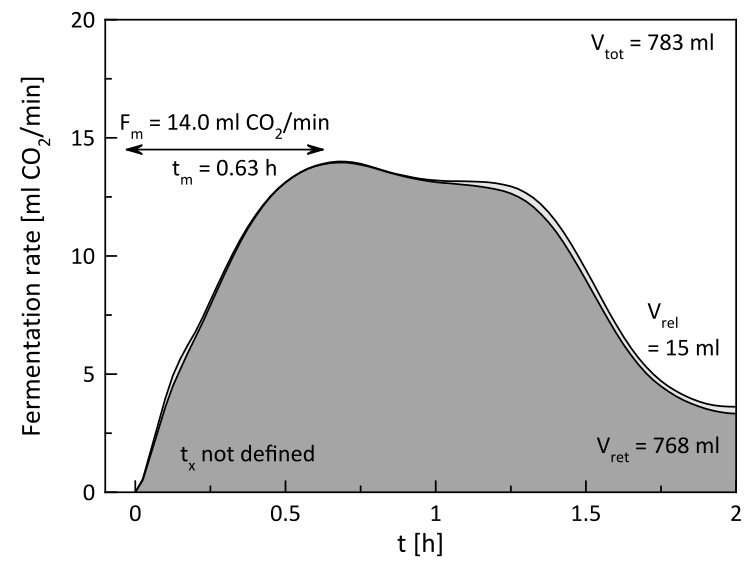

(b)

Figure 6: The gaseous release curves for fermenting dough prepared with (a) 6 wt\% sugar, no salt; (b) no sugar and $1.5 \mathrm{wt} \%$ salt; (c) $6 \mathrm{wt} \%$ sugar, $1.5 \mathrm{wt} \%$ salt. The area shaded in dark grey represents the amount of $\mathrm{CO}_{2}$ retained within the dough, whereas the area shaded in light grey stands for the amount of $\mathrm{CO}_{2}$ released by the dough. The time $t_{m}$ denotes the instant at which the maximum fermentation rate $F_{m}$ is reached, whereas $t_{x}$ represents the instant at which the dough starts to release gas. 


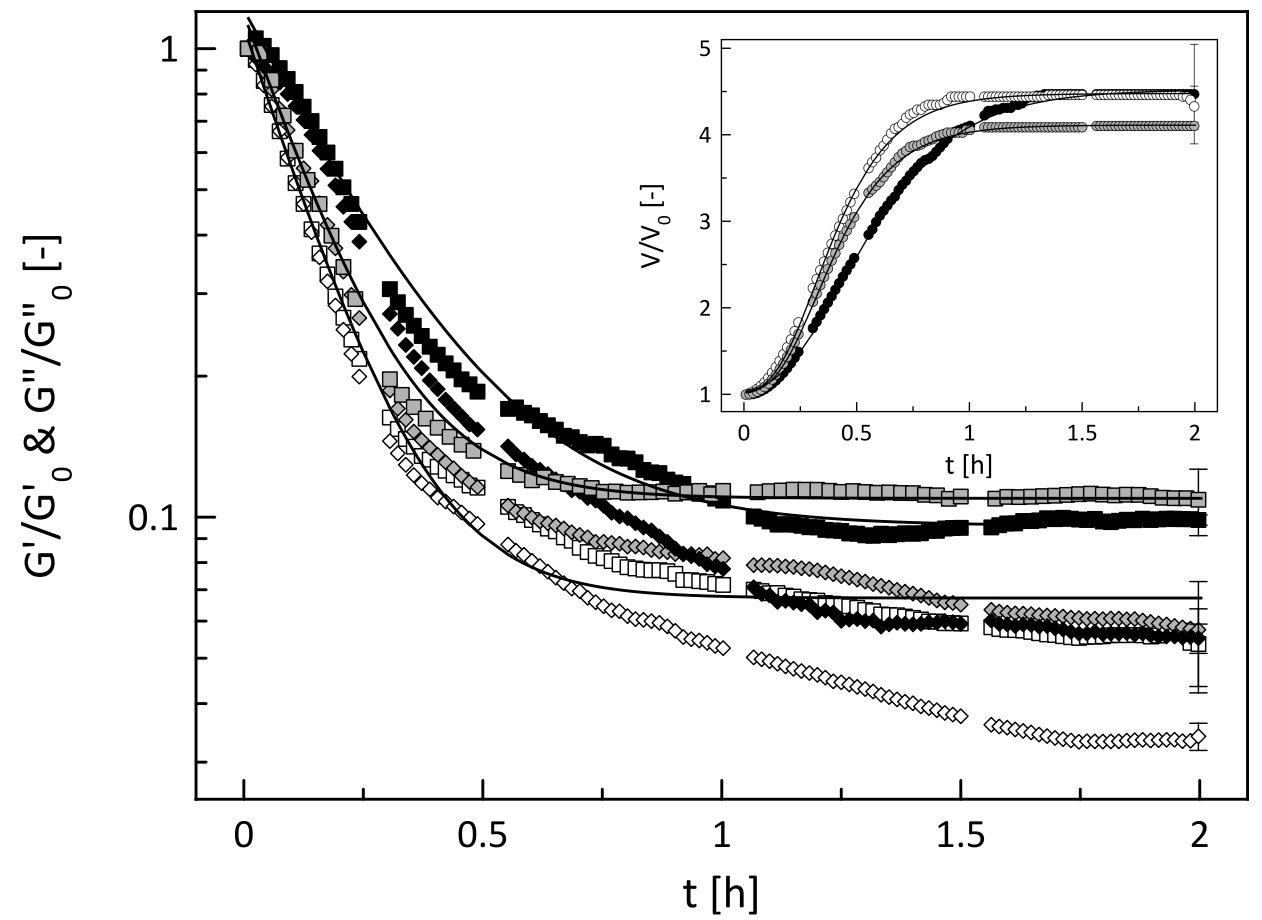

Figure 7: The decrease in the rescaled dynamic moduli $G^{\prime}(t)$ (squares) and $G^{\prime \prime}(t)$ (diamonds) after normalisation with respect to their initial values $G_{0}^{\prime}$ and $G_{0}^{\prime \prime}$ for fermenting dough prepared with $6 \mathrm{wt} \%$ sugar and no salt (white); no sugar and $1.5 \mathrm{wt} \%$ salt (grey); $6 \mathrm{wt} \%$ sugar and $1.5 \mathrm{wt} \%$ salt (black). Inset: the corresponding increase in volume $V(t)$ after normalisation with respect to the initial volume $V_{0}$ for the three conditions. The time evolution of $G^{\prime}(t) / G_{0}^{\prime}$ and $V(t) / V_{0}$ can be described by an exponential decay function (eq. 9) and by the modified Gompertz model (eq. 10), respectively, as indicated by the black lines. All measurements were performed in triplicate. For the sake of clarity, error bars indicating the standard deviation are only shown for the final data points. 


\section{${ }_{882}$ List of Tables}

883 1 Fitting parameters for the exponential decay function describing $G^{\prime}(t) / G_{0}^{\prime}$ 
Table 1: Fitting parameters for the exponential decay function (eq. 9) describing the relative decrease in the storage modulus $G^{\prime}(t) / G_{0}^{\prime}$ and for the modified Gompertz model (eq. 10) describing the relative increase in volume $V(t) / V_{0}$ for fermenting dough prepared with different concentrations of salt and sugar. The $R^{2}$ values for curve fitting were always higher than 0.992 .

\begin{tabular}{l|ccc|ccc}
\hline \multicolumn{9}{c}{$G^{\prime}(t) / G_{\mathbf{0}}^{\prime}$} & \multicolumn{3}{c}{$V(t) / V_{\mathbf{0}}$} \\
\hline & $G_{0}^{\prime}[\mathrm{Pa}]$ & $G_{\mathrm{e}}^{\prime}[\mathrm{Pa}]$ & $t_{\mathrm{d}}[\mathrm{s}]$ & $b_{1}[-]$ & $b_{2}\left[\mathrm{~s}^{-1}\right]$ & $t_{\text {lag }}[\mathrm{s}]$ \\
\hline 6\% sugar 0\% salt & $3630 \pm 218$ & $243 \pm 15$ & $476 \pm 4$ & $3.477 \pm 0.004$ & $0.0019 \pm 1 \cdot 10^{-5}$ & $473 \pm 7$ \\
$0 \%$ sugar 1.5\% salt & $4598 \pm 641$ & $506 \pm 65$ & $524 \pm 5$ & $3.111 \pm 0.002$ & $0.0017 \pm 7 \cdot 10^{-6}$ & $494 \pm 4$ \\
$6 \%$ sugar 1.5\% salt & $3677 \pm 479$ & $355 \pm 46$ & $770 \pm 12$ & $3.523 \pm 0.004$ & $0.0013 \pm 7 \cdot 10^{-6}$ & $576 \pm 7$ \\
\hline
\end{tabular}

